\title{
The diversity of endolichenic fungi - A review
}

\author{
Jyotsna Chakarwarti ${ }^{1,2}$, Sanjeeva Nayaka ${ }^{1,2^{*}}$ and Suchi Srivastava $a^{2,3}$ \\ ${ }^{1}$ Lichenology Laboratory, CSIR-National Botanical Research Institute, Rana Pratap Marg, Lucknow-226001, India \\ ${ }^{2}$ Academy of Scientific and Innovative Research (AcSIR), CSIR-HRDC Campus, Kamla Nehru Nagar, Ghaziabad - \\ 201002, India \\ ${ }^{3}$ Plant Ecology and Environmental Technologies Division, CSIR-National Botanical Research Institute, Rana Pratap \\ Marg, Lucknow-226001, India
}

Chakarwarti J, Nayaka S, Srivastava S 2020 - Diversity of endolichenic fungi - A review. Asian Journal of Mycology 3(1), 488-509, Doi 10.5943/ajom/3/1/18

\begin{abstract}
Endolichenic fungi reside inside the lichen thalli without showing any visible symptoms of their presence. The fossil record of endolichenic fungi dates back to 415 million years, however, they have been successfully isolated only since the 1990s. Several reviews are available dealing with the bioprospecting potential of unique metabolites produced by endolichenic fungi; however, reviews addressing their taxonomic diversity are lacking. In this review, the names and the current taxonomic status of the endolichenic fungi reported so far are compiled and updated. The study indicated that approximately 500 endolichenic fungi are isolated of which only 135 identified up to species level. A large number of endolichenic fungi are either partially identified up to genus level or treated as unidentified. The reasons for such treatment are discussed. The study also revealed that only 114 well-known species of lichens belonging to 22 families are utilized for isolation of endolichenic fungi, while in some cases hosts were not identified. These lichens belong mostly to macro lichens with Parmeliaceae as the dominant members. The occurrence of the endolichenic fungi on different groups of plants indicates that they are not host-specific.
\end{abstract}

Keywords - Endosymbionts - lichenicolous fungi - miniature ecosystem - symbiosis

\section{Introduction}

Lichens are considered as a miniature ecosystem (Honegger 1991) due to their close interaction with a variety of organisms such as fungi, algae, cyanobacteria, eubacteria, and microinvertebrates. While a fungus (Ascomycetes or Basidiomycetes) and a photoautotroph (Chlorophyta or Cyanobacteria) make a primary association and form lichen thallus, others make secondary, obligate or opportunistic associations. Recently, yeasts thought to be obligate, third partners in lichen formation (Spribille et al. 2016), however, this claim is not yet accepted (Mark et al. 2020). Due to growing ambiguity over the presence of yeast and other microbes, the lichens are redefined as 'a self-sustaining ecosystem formed by the interaction of an exhabitant fungus and an extracellular arrangement of one or more photosynthetic partners and an indeterminate number of other microscopic organisms' (Hawksworth \& Grube 2020). Among the secondary associates, the lichenicolous and endolichenic fungi (hereafter referred to as ELF) are interesting groups due to their taxonomic and evolutionary status (Oh et al. 2020), and for bioprospecting (Maduranga et al. 2018). The ELF, which are endosymbionts of lichens (Miadlikowska et al. 2004) are predominantly filamentous, reside inside the lichen thallus without showing any visible symptoms of their 
presence and apparently, do not harm the host. The ELF closely associates themselves with the photobiont of the lichen for their nutrition (Arnold et al. 2009). They are similar to endophytic fungi of vascular plants. The endophytic fungi play various functional roles in their plant hosts like altering their disease resistance or susceptibility, augmenting their growth, and ability to survive in extreme environments ( $\mathrm{Yu}$ et al. 2018). However, the role of endolichenic fungi in increasing the fitness of lichens is not known (Suryanarayan et al. 2017).

\section{Diversity of ELF}

The ELF are cosmopolitan in distribution and are known from every lichen species tested from the Arctic to tropics. Around 13,500 species of ELF are estimated to occur in the world (Kellogg \& Raja 2016). The ELF exhibits a vast diversity as indicated by Petrini et al. (1990), who isolated 506 fungal taxa from 17 lichen species; of these, 306 were isolated only once. An intensive study of two lichens Peltigera praetextata and Xanthoparmelia taractica from a coniferous forest (Aosta Valley, Italy) yielded different fungi belonging to 117 taxa (Girlanda et al. 1997). Suryanarayanan et al. (2005) isolated 242 endolichenic fungi from 500 thallus segments of five corticolous lichens. Li et al. (2007) isolated a total of 32 taxa from 488 thallus segments, with 31 ascomycetes and one yeast. Zhang et al. (2016) recovered 247 fungal isolates from 2712 thallus segments of 17 lichen species. Govinda Rajulu et al. (2020) isolated a total of 389 species from 10 lichen species of Western Ghats, Southern India. These reports exemplify the tremendous diversity of ELF. Rashmi et al. (2019) provided a lengthy list of endophytic fungi reported from the world, however, it included very few ELFs (e.g. Nodulisporium hyalosporum). The compilation of all the ELF studies carried out so far indicated that about 500 ELFs have been isolated till date, among them only 135 were identified up to species level while most of them are either identified up to the genus or class level or treated as unidentified (Table 1). As the ELFs do not produce sufficient morphological characters such as colouration, conidia or spores in culture, it is difficult to identify under routine microscopic studies (Tripathi \& Joshi 2019). The DNA based molecular techniques are only partially helpful due to the lack of sequences in gene bank or library related to ELFs. Usullay, the aim of most of the researchers is to isolate ELFs for bioactive molecule and hence not much attention is paid towards their taxonomy. Recent studies have indicated that ELFs are not different from endophytes and the DNA based techniques are able to unravel their identity (Govinda Rajulu et al. 2020).

Table 1 List of lichens so far explored for ELFs (name in parenthesis are synonyms as originally annotated by authors)

\begin{tabular}{|c|c|c|c|}
\hline & Fungi & Host lichen & Reference \\
\hline \multirow[t]{6}{*}{1} & \multirow[t]{6}{*}{ Acremonium sp. } & Cladonia sp.; Stereocaulon sp. & Petrini et al. (1990) \\
\hline & & Parmotrema thomsonii (= Parmelia thomsonii) & Suryanarayanan et al. (2005) \\
\hline & & $\begin{array}{l}\text { Dermatocarpon miniatum; Melanelia sorediata; } \\
\text { Parmotrema thomsonii }\end{array}$ & Li et al. (2007) \\
\hline & & Parmotrema sp. & Kannangara et al. (2008) \\
\hline & & $\begin{array}{l}\text { Lobaria kurokawae; Parmotrema thomsonii; } \\
\text { P. tinctorum; Punctelia rudecta }\end{array}$ & Tripathi et al. (2014) \\
\hline & & $\begin{array}{l}\text { Lobaria kurokawae; Parmotrema tinctorum; } \\
\text { Punctelia rudecta }\end{array}$ & Suryanarayanan et al. (2017) \\
\hline \multirow[t]{3}{*}{2} & \multirow{3}{*}{$\begin{array}{l}\text { Alternaria } \mathrm{sp} . \\
(=\text { Ulocladium } \mathrm{sp} .)\end{array}$} & Peltigera praetextata; Xanthoparmelia taractica & Girlanda et al. (1997) \\
\hline & & Parmotrema hababianum & Suryanarayanan et al. (2017) \\
\hline & & Hypotrachyna sp.; Unknown lichen & Wang et al. (2012) \\
\hline 3 & $\begin{array}{l}\text { A. alternariae (Cooke) } \\
\text { Woudenb. \& Crous } \\
\text { (= Ulocladium alternariae } \\
\text { (Cooke) Simmons) }\end{array}$ & $\begin{array}{l}\text { Xanthoparmelia taractica }(=\text { Parmelia taractica }) \\
\text { Peltigera praetextata }\end{array}$ & Girlanda et al. (1997) \\
\hline \multirow[t]{2}{*}{4} & \multirow[t]{2}{*}{ A. alternata (Fr.) Keissl. } & Peltigera praetextata; Xanthoparmelia taractica & Girlanda et al. (1997) \\
\hline & & $\begin{array}{l}\text { Bulbothrix meizospora; Heterodermia flabellata; } \\
\text { H. hypochraea; Parmotrema crinitum; }\end{array}$ & Suryanarayanan et al. (2005) \\
\hline
\end{tabular}


Table 1 Continued.

\begin{tabular}{|c|c|c|c|}
\hline & Fungi & Host lichen & Reference \\
\hline & & $\begin{array}{l}\text { P. praesorediosum; } P \text {. nilgherrense; } \\
P \text {. reticulatum; } P \text {. thomsonii; } \text { Physcia dilatate }\end{array}$ & \\
\hline & & $\begin{array}{l}\text { Heterodermia flabellata; Parmotrema crinitum; } \\
\text { P. thomsonii; Physcia dilatate }\end{array}$ & Li et al. (2007) \\
\hline & & Usnea aciculifera & He et al. (2012) \\
\hline & & $\begin{array}{l}\text { Bulbothrix meizospora; Heterodermia flabellata; } \\
\text { H. hypochraea; Flavoparmelia caperata; } \\
\text { Leptogium burnetiae; Parmotrema critinum; } \\
\text { P. graynum; } \text { P. nilgherrense; } \text { P. praesorediosum; } \\
\text { P. reticulatum; P. thomsonii; Physcia dilatate }\end{array}$ & Tripathi et al. (2014) \\
\hline & & $\begin{array}{l}\text { Cladonia fruticulosa; Parmotrema reticulatum; } \\
\text { P. tinctorum }\end{array}$ & Vinayaka et al. (2016) \\
\hline & & \#Not specified - list of several lichens given & Zhang et al. (2016) \\
\hline 5 & $\begin{array}{l}\text { A. tenuissima (Kunze) } \\
\text { Wiltshire }\end{array}$ & Cladonia sp.; Stereocaulon sp. & Petrini et al. (1990) \\
\hline 6 & Aphanocladium sp. & Cladonia sp.; Stereocaulon sp. & Petrini et al. (1990) \\
\hline 7 & Apiosordaria sp. & \#Not specified - list of several lichens given & Zhang et al. (2016) \\
\hline 8 & $\begin{array}{l}\text { Apiotrichum } \\
\text { (= Hyalodendron sp.) }\end{array}$ & Cladonia sp.; Stereocaulon sp. & Petrini et al. (1990) \\
\hline 9 & $\begin{array}{l}\text { Arthrinium arundinis (corda) } \\
\text { Dyko \& B. Sutton (= } \\
\text { Apiospora montagnei Sacc.) }\end{array}$ & Cladonia sp. & Wang et al. (2017) \\
\hline \multirow[t]{2}{*}{10} & \multirow{2}{*}{$\begin{array}{l}\text { Arthrinium phaeospermum } \\
\text { (Corda) M. B. Ellis }\end{array}$} & Cladonia sp.; Stereocaulon sp. & Petrini et al. (1990) \\
\hline & & Peltigera praetextata; Xanthoparmelia taractica & Girlanda et al. (1997) \\
\hline 11 & Ascochyta sp. & Cladonia sp.; Stereocaulon sp. & Petrini et al. (1990) \\
\hline \multirow[t]{6}{*}{12} & \multirow{6}{*}{$\begin{array}{l}\text { Aspergillus } \mathrm{sp} . \\
(=\text { Eurotium } \mathrm{sp} .)\end{array}$} & Pseudocyphellaria $\mathrm{sp}$. & Kannangara et al. (2008) \\
\hline & & Peltigera elisabethae var. mauritzii & Zhao et al. (2014) \\
\hline & & Cetrelia sp. & $\begin{array}{l}\text { Chen et al. (2014), Chen et al. } \\
(2019)\end{array}$ \\
\hline & & Cladina grisea & Chen et al. (2014) \\
\hline & & Parmelia sp. & $\begin{array}{l}\text { Padhi and Tayung }(2015, \\
2016)\end{array}$ \\
\hline & & Phaeophyscia hispidula & Suryanarayanan et al. (2017) \\
\hline 13 & A. aculeatus Iizuka & Porina tetracerae & Maduranga et al. (2018) \\
\hline 14 & $\begin{array}{l}\text { A. coremiiformis Bartoli \& } \\
\text { Maggi }\end{array}$ & Flavoparmelia caperata & Tripathi et al. (2014) \\
\hline \multirow[t]{3}{*}{15} & \multirow[t]{3}{*}{ A. flavus Link } & $\begin{array}{l}\text { Bulbothrix meizospora; Heterodermia flabellata; } \\
\text { H. hypochraea; Parmotrema crinitum; } \\
\text { P. nilgherrense; P. praesorediosum; } \\
\text { P. reticulatum; P. thomsonii; Physcia dilatate }\end{array}$ & Suryanarayanan et al. (2005) \\
\hline & & $\begin{array}{l}\text { Heterodermia flabellata; Parmotrema crinitum; } \\
\text { P. thomsonii; Physcia dilatate }\end{array}$ & Li et al. (2007) \\
\hline & & $\begin{array}{l}\text { Bulbothrix meizospora; Flavoparmelia caperata; } \\
\text { Heterodermia flabellata; } \text { H. hypochraea; } \\
\text { Leptogium burnetiae; Parmotrema crinitum; } \\
\text { P. graynum; } \text { P. nilgherrense; P. praesorediosum; } \\
\text { P. reticulatum; P. thomsonii; Physcia dilatate }\end{array}$ & Tripathi et al. (2014) \\
\hline 16 & A. fumigatus Fresen. & Unidentified & Maduranga et al. (2018) \\
\hline 17 & $\begin{array}{l}\text { A. hiratsukae Udagawa, } \\
\text { Tsub. \& Y. Horie }\end{array}$ & $\begin{array}{l}\text { Opegrapha medusulina; Pyxine cocoes; Roccella } \\
\text { montagnei; Unidentified }\end{array}$ & Maduranga et al. (2018) \\
\hline \multirow[t]{4}{*}{18} & \multirow[t]{4}{*}{ A. niger Tiegh. } & $\begin{array}{l}\text { Heterodermia flabellata; Parmotrema thomsonii; } \\
\text { Physcia dilatata; Roccella montagnei }\end{array}$ & Suryanarayanan et al. (2005) \\
\hline & & $\begin{array}{l}\text { Heterodermia flabellata; Parmotrema thomsonii; } \\
\text { Physcia dilatate }\end{array}$ & $\begin{array}{l}\text { Li et al. (2007), Tripathi et al. } \\
(2014)\end{array}$ \\
\hline & & $\begin{array}{l}\text { Parmotrema cristiferum; Ramalina arabum; } \\
\text { Teloschistes flavicans; Usnea galbinifera }\end{array}$ & Vinayaka et al. (2016) \\
\hline & & Parmotrema ravum & Padhi et al. (2019) \\
\hline
\end{tabular}


Table 1 Continued.

\begin{tabular}{|c|c|c|c|}
\hline & Fungi & Host lichen & \begin{tabular}{r|} 
Reference \\
\end{tabular} \\
\hline \multirow[t]{3}{*}{19} & \multirow[t]{3}{*}{ A. versicolor (Vuill.) Tirab. } & Lobaria retigera & Duo et al. (2014) \\
\hline & & Ricasolia quercizans (= Lobaria quercizans) & Li et al. (2015a) \\
\hline & & Unknown & Li et al. (2015a) \\
\hline 20 & A. quadricinctus E. Yuill & Usnea longissima & Prateeksha et al. (2020) \\
\hline \multirow[t]{2}{*}{21} & \multirow[t]{2}{*}{$\begin{array}{l}\text { Aureobasidium pullulans (de } \\
\text { Bary \& Löwenthal) G. } \\
\text { Arnaud }\end{array}$} & $\begin{array}{l}\text { Canoparmelia texana; Heterodermia diademata; } \\
\text { H. podocarpa; Leptogium askotense; Lobaria } \\
\text { kurokawae; Parmotrema hababianum; } \\
\text { P. tinctorum; Phaeophyscia hispidula; Punctelia } \\
\text { rudecta; Ramalina conduplicans; Usnea sp. }\end{array}$ & Suryanarayanan et al. (2017) \\
\hline & & Bacidina sp.; Teloschistes flavicans & Govinda Rajulu et al. (2020) \\
\hline 22 & Bahusakala sp. & Cladonia sp.; Stereocaulon sp. & Petrini et al. (1990) \\
\hline 23 & $\begin{array}{l}\text { Berkeleyomyces basicola } \\
\text { (Berk. \& Broome) W.J. Nel, } \\
\text { Z.W. de Beer, T.A. Duong \& } \\
\text { M.J. Wingf (= Thielavia } \\
\text { basicola (Berk. \& Broome) } \\
\text { Zopf) }\end{array}$ & $\begin{array}{l}\text { Polyblastidium dendriticum }(=\text { Heterodermia } \\
\text { dendritica }) ; \text { Parmotrema reticulatum } ; \text { Ramalina } \\
\text { arabum; Usnea galbinifera }\end{array}$ & Vinayaka et al. (2016) \\
\hline 24 & Biatriospora sp. & Pseudocyphellaria sp. & Zhou et al. (2016) \\
\hline 25 & $\begin{array}{l}\text { Biscogniauxia petrensis Z.F. } \\
\text { Zhang, F. Liu \& L. Cai }\end{array}$ & Parmotrema sp. & Oh et al. (2020) \\
\hline 26 & Bispora sp. & Canoparmelia texana & Suryanarayanan et al. (2017) \\
\hline \multirow[t]{3}{*}{27} & \multirow[t]{3}{*}{ Botrytis sp. } & Roccella montagnei & Suryanarayanan et al. (2005) \\
\hline & & Parmotrema tinctorum & Suryanarayanan et al. (2017) \\
\hline & & Dirinaria consimilis & Govinda Rajulu et al. (2020) \\
\hline 28 & B. cinerea Pers. & Cladonia sp.; Stereocaulon sp. & Petrini et al. (1990) \\
\hline 29 & Broomella sp. & Parmotrema sp. & Kannangara et al. (2008) \\
\hline 30 & $\begin{array}{l}\text { Byssochlamys spectabilis } \\
\text { (Udagawa \& Shoji Suzuki) } \\
\text { Houbraken \& Samson }\end{array}$ & Pyrenula ochraceoflava & Maduranga et al. (2018) \\
\hline 31 & $\begin{array}{l}\text { Cadophora bubakii (Laxa) } \\
\text { Damm \& S. Bien } \\
\text { (= Phailophora bubakii } \\
\text { (Laxa) Schol-Schwarz) }\end{array}$ & $\begin{array}{l}\text { Dermatocarpon miniatum; Melanelia sorediata; } \\
\text { Ramalina sinensis }\end{array}$ & Li et al. (2007) \\
\hline 32 & $\begin{array}{l}\text { Calonectria gracilis Corus, } \\
\text { M.J. Wingf. \& Alfenaas } \\
\text { (= Cylindrocarpon gracile } \\
\text { Bugnic.) }\end{array}$ & Peltigera praetextata; Xanthoparmelia taractica & Girlanda et al. (1997) \\
\hline 33 & Cerrena sp. & Pyrenocarp sp.; Pyrenula indet & Maduranga et al. (2018) \\
\hline 34 & $\begin{array}{l}\text { Cerrena unicolor (Bull.) } \\
\text { Murrill }\end{array}$ & Roccella montagnei & Maduranga et al. (2018) \\
\hline 35 & Chaetomella sp. & Parmotrema nilgherrense & $\begin{array}{l}\text { Suryanarayanan et al. (2005), } \\
\text { Tripathi et al. (2014) }\end{array}$ \\
\hline \multirow[t]{5}{*}{36} & \multirow[t]{5}{*}{ Chaetomium sp. } & Roccella montagnei & Suryanarayanan et al. (2005) \\
\hline & & Punctelia borreri & Li et al. (2007) \\
\hline & & $\begin{array}{l}\text { Canoparmelia texana; Heterodermia diademata; } \\
\text { H. podocarpa; Leptogium askotense; Lobaria } \\
\text { kurokawae; Parmotrema tinctorum; Phaeophyscia } \\
\text { hispidula; Punctelia rudecta }\end{array}$ & Suryanarayanan et al. (2017) \\
\hline & & $\begin{array}{l}\text { Heterodermia incana; Parmotrema cristiferum; } \\
\text { P. reticulatum; } P \text {. tinctorum; Ramalina arabum; } \\
\text { Ramalina pacifica; Teloschistes flavicans }\end{array}$ & Vinayaka et al. (2016) \\
\hline & & $\begin{array}{l}\text { Bacidina sp.; Dirinaria consimilis; Evernia } \\
\text { prunastri; Parmotrema sp.; Usnea } \mathrm{sp} .\end{array}$ & Govinda Rajulu et al. (2020) \\
\hline \multirow[t]{3}{*}{37} & \multirow[t]{3}{*}{ C. elatum Kunze } & Melanelia sorediata & Li et al. (2007) \\
\hline & & Hypotrachyna cirrhata $(=$ Everniastrum cirrhatum $)$ & Chen et al. (2013) \\
\hline & & $\begin{array}{l}\text { Parmotrema reticulatum; Ramalina arabum; Usnea } \\
\text { galbinifera }\end{array}$ & Vinayaka et al. (2016) \\
\hline
\end{tabular}


Table 1 Continued.

\begin{tabular}{|c|c|c|c|}
\hline & Fungi & Host lichen & $\begin{array}{r}\text { Reference } \\
\end{array}$ \\
\hline \multirow[t]{4}{*}{38} & \multirow[t]{4}{*}{ C. globosum Kunze } & Cladonia coniocraea; Melanelia sorediata & Li et al. (2007) \\
\hline & & $\begin{array}{l}\text { Hypotrachyna nepalensis (= Everniastrum } \\
\text { nepalense) }\end{array}$ & Zheng et al. (2014) \\
\hline & & $\begin{array}{l}\text { Cladonia fruticulosa; Parmotrema cristiferum; } \\
\text { P. reticulatum; P. tinctorum; Polyblastidium } \\
\text { dendriticum; Ramalina pacifica; Usnea } \\
\text { stigmatoides }\end{array}$ & Vinayaka et al. (2016) \\
\hline & & \#Not specified - list of several lichens given & Zhang et al. (2016) \\
\hline \multirow[t]{2}{*}{39} & \multirow[t]{2}{*}{ Chalara sp. } & Cladonia sp.; Stereocaulon sp. & Petrini et al. (1990) \\
\hline & & $\begin{array}{l}\text { Parmotrema reticulatum; Ramalina arabum; Usnea } \\
\text { stigmatoides }\end{array}$ & Vinayaka et al. (2016) \\
\hline 40 & $\begin{array}{l}\text { C. constricta NagRaj \& } \\
\text { W.B. Kendr. }\end{array}$ & Cladonia sp.; Stereocaulon sp. & Petrini et al. (1990) \\
\hline 41 & $\begin{array}{l}\text { C. fusidioides (Corda) } \\
\text { Rabenh. }\end{array}$ & Cladonia sp.; Stereocaulon sp. & Petrini et al. (1990) \\
\hline 42 & Chrysosporium sp. & Parmotrema sp.; Usnea sp. & Kannangara et al. (2008) \\
\hline 43 & $\begin{array}{l}\text { C. pseudomerdarium van } \\
\text { Oorschot }\end{array}$ & Cladonia sp.; Stereocaulon sp. & Petrini et al. (1990) \\
\hline \multirow[t]{2}{*}{44} & \multirow{2}{*}{$\begin{array}{l}\text { Cladophialophora } \\
\text { chaetospira (Grove) Crous } \\
\text { \& Arzanlou (= } \\
\text { Heteroconium chaetospira } \\
\text { (Grove) M. B. Ellis) }\end{array}$} & Cladonia sp.; Stereocaulon sp. & Petrini et al. (1990) \\
\hline & & Peltigera praetextata; Xanthoparmelia taractica & Girlanda et al. (1997) \\
\hline \multirow[t]{7}{*}{45} & \multirow[t]{7}{*}{ Cladosporium sp. } & Cladonia sp.; Stereocaulon sp. & Petrini et al. (1990) \\
\hline & & $\begin{array}{l}\text { Roccella montagnei; Parmotrema nilgherrense; } \\
\text { P. praesorediosum; Physcia dilatata }\end{array}$ & Suryanarayanan et al. (2005) \\
\hline & & Physcia dilatata & Li et al. (2007) \\
\hline & & Pseudocyphellaria sp.; Parmotrema sp.; Usnea sp. & Kannangara et al. (2008) \\
\hline & & $\begin{array}{l}\text { Parmotrema hababianum; P. nilgherrense; } \\
\text { P. praesorediosum; Lobaria kurokawae; Physcia } \\
\text { dilatata }\end{array}$ & Tripathi et al. (2014) \\
\hline & & Lobaria kurokawae & Suryanarayanan et al. (2017) \\
\hline & & $\begin{array}{l}\text { Arthonia sp.; Hypotrachyna crenata; Parmotrema } \\
\text { tinctorum }\end{array}$ & Govinda Rajulu et al. (2020) \\
\hline \multirow[t]{3}{*}{46} & \multirow{3}{*}{$\begin{array}{l}\text { C. cladosporioides }(\mathrm{Fr} .) \mathrm{de} \\
\text { Vries }\end{array}$} & Cladonia sp.; Stereocaulon sp. & Petrini et al. (1990) \\
\hline & & Peltigera praetextata; Xanthoparmelia taractica & Girlanda et al. (1997) \\
\hline & & $\begin{array}{l}\text { Heterodermia incana; Ramalina arabum; } \\
\text { Teloschistes flavicans; Usnea stigmatoides; } \\
\text { U. galbinifera }\end{array}$ & Vinayaka et al. (2016) \\
\hline 47 & C. herbarum (Pers.) Link & Cladonia sp.; Stereocaulon sp. & Petrini et al. (1990) \\
\hline 48 & Colletotrichum sp. & Cladonia sp.; Stereocaulon sp. & Petrini et al. (1990) \\
\hline 49 & C. dematium (Pers.) Grove & $\begin{array}{l}\text { Parmotrema tinctorum; Polyblastidium } \\
\text { dendriticum; Ramalina arabum; } \text { R. pacifica; } \\
\text { Usnea stigmatoides }\end{array}$ & Vinayaka et al. (2016) \\
\hline \multirow[t]{4}{*}{50} & \multirow[t]{4}{*}{$\begin{array}{l}\text { Coniochaeta sp. }(= \\
\text { Lecythophora } \mathrm{sp} .)\end{array}$} & $\begin{array}{l}\text { Dermatocarpon miniatum; Parmelia } \mathrm{sp} . ; \text { Punctelia } \\
\text { borreri; Zeroviella mandschurica (= Xanthoria } \\
\text { mandschurica) }\end{array}$ & Li et al. (2007) \\
\hline & & Zeroviella mandschurica & Wang et al. (2010) \\
\hline & & \#Not specified - list of several lichens given & Zhang et al. (2016) \\
\hline & & Cladonia evansii & Wijeratne et al. (2016) \\
\hline \multirow[t]{2}{*}{51} & \multirow[b]{2}{*}{$\begin{array}{l}\text { Cordyceps farinose } \\
\text { (Holmsk.) Kepler, B. } \\
\text { Shrestha \& Spatafora } \\
\text { (= Paecilomyces farinosus } \\
\text { (Holm ex S. F. Gray) Brown } \\
\text { et Smith) }\end{array}$} & Cladonia sp.; Stereocaulon sp. & Petrini et al. (1990) \\
\hline & & Peltigera praetextata; Xanthoparmelia taractica & Girlanda et al. (1997) \\
\hline 52 & Corynespora sp. & Usnea cavernosa & Paranagama et al. (2007) \\
\hline
\end{tabular}


Table 1 Continued.

\begin{tabular}{|c|c|c|c|}
\hline & Fungi & Host lichen & Reference \\
\hline 53 & $\begin{array}{l}\text { Cosmospora butyri (J.F.H. } \\
\text { Beyma) Gräfenhan, Seifert } \\
\text { \& Schroers (=Acremonium } \\
\text { butyri (Beyma) W. Gams) }\end{array}$ & Peltigera praetextata; Xanthoparmelia taractica & Girlanda et al. (1997) \\
\hline 54 & Cryptocline sp. & Cladonia sp.; Stereocaulon sp. & Petrini et al. (1990) \\
\hline 55 & C. fasciculata (Tode) Petr. & Cladonia sp.; Stereocaulon sp. & Petrini et al. (1990) \\
\hline 56 & Curvularia sp. & Pseudocyphellaria sp.; Usnea sp. & Kannagara et al. (2008) \\
\hline 57 & $\begin{array}{l}\text { C. australiensis (Bugnic. ex } \\
\text { M.B. Ellis) Manamgoda, L. } \\
\text { Cai \& K.D. Hyde } \\
\text { (= Bipolaris australiensis } \\
\text { (Bugnic. ex M.B. Ellis) } \\
\text { Tsuda \& Ueyama) }\end{array}$ & Heterodermia flabellata; Physcia dilatata & $\begin{array}{l}\text { Suryanarayanan et al. (2005), } \\
\text { Li et al. (2007), Tripathi et al. } \\
(2014)\end{array}$ \\
\hline 58 & C. lunata (Wakker) Boedijn & $\begin{array}{l}\text { Polyblastidium dendriticum; Ramalina pacifica; } \\
\text { Usnea stigmatoides }\end{array}$ & Vinayaka et al. (2016) \\
\hline 59 & \begin{tabular}{|l} 
C. spicifera (Bainier) \\
Boedijn (= Drechslera \\
anamorph Cochliobolus \\
spicifer Nelson)
\end{tabular} & Peltigera praetextata; Xanthoparmelia taractica & Girlanda et al. (1997) \\
\hline 60 & $\begin{array}{l}\text { C. trifolii (Kauffman) } \\
\text { Boedijn }\end{array}$ & Usnea sp. & Samanthi et al. (2015a) \\
\hline 61 & $\begin{array}{l}\text { Cylindromonium lichenicola } \\
\text { (W. Gams) Crous } \\
\text { (=Acremonium lichenicola } \\
\text { W. Gams) }\end{array}$ & Parmotrema reticulatum & $\begin{array}{l}\text { Suryanarayanan et al. (2005), } \\
\text { Tripathi et al. (2014) }\end{array}$ \\
\hline 62 & Cylindrosporium sp. & Bulbothrix meizospora & $\begin{array}{l}\text { Suryanarayanan et al. (2005), } \\
\text { Tripathi et al. (2014) }\end{array}$ \\
\hline 63 & $\begin{array}{l}\text { Cystobasidium laryngis } \\
\text { (Reiersöl) Yurkov }\end{array}$ & \#Not specified - list of several lichens given & Zhang et al. (2016) \\
\hline 64 & Cytospora sp. & Parmelia sp. & Padhi and Tayung (2015) \\
\hline 65 & Daldinia sp. & Dirinaria picta; Pyxine cocoes & Maduranga et al. (2018) \\
\hline \multirow[t]{3}{*}{66} & \multirow[t]{3}{*}{$\begin{array}{l}\text { Daldinia eschscholtzii } \\
\text { (Ehrenb.) Rehm }\end{array}$} & $\begin{array}{l}\text { Dirinaria picta; Opegrapha medusulina; } \\
\text { O. arabica; Porina tetracerae; Roccella montagnei }\end{array}$ & Maduranga et al. (2018) \\
\hline & & Dirinaria consimilis & Govinda Rajulu et al. (2020) \\
\hline & & Leptogium $\mathrm{sp}$. & Oh et al. (2020) \\
\hline 67 & $\begin{array}{l}\text { Diaporthe sp. (= Phomopsis } \\
\text { sp.) }\end{array}$ & Cladonia sp.; Porina tetracerae; Stereocaulon sp. & $\begin{array}{l}\text { Petrini et al. (1990), Padhi \& } \\
\text { Tayung (2015), Maduranga et } \\
\text { al. (2018) }\end{array}$ \\
\hline 68 & $\begin{array}{l}\text { D. arengae R.R. Gomes, } \\
\text { Glienke \& Crous }\end{array}$ & $\begin{array}{l}\text { Arthonia antillarum; Opegrapha medusulina; } \\
\text { Unidentified }\end{array}$ & Maduranga et al. (2018) \\
\hline 69 & Endomelanconiopsis sp. & Porina tetracerae; Pyrenula $\mathrm{sp}$. & Maduranga et al. (2018) \\
\hline 70 & $\begin{array}{l}\text { E. endophytica E.I. Rojas \& } \\
\text { Samuels }\end{array}$ & Porina tetracerae; Pyrenula sp.; Unidentified & Maduranga et al. (2018) \\
\hline 71 & $\begin{array}{l}\text { Epicoccum nigrum Link }(= \\
\text { Epicoccum purpurascens } \\
\text { Ehrenb. ex Schlecht) }\end{array}$ & Cladonia sp.; Stereocaulon sp. & Petrini et al. (1990) \\
\hline 72 & Exophiala sp. & Cladonia sp.; Stereocaulon sp. & Petrini et al. (1990) \\
\hline 73 & $\begin{array}{l}\text { E. lecanii-corni (Benedek \& } \\
\text { G. Specht) Haase \& de } \\
\text { Hoog, (=E. jeanselmei var. } \\
\text { lecanii-corvi (Benedeck \& } \\
\text { Specht) De Hoog) }\end{array}$ & Cladonia sp.; Stereocaulon sp. & Petrini et al. (1990) \\
\hline 74 & $\begin{array}{l}\text { E. mansonii (Castell.) De } \\
\text { Hoog }\end{array}$ & Cladonia sp.; Stereocaulon sp. & Petrini et al. (1990) \\
\hline \multirow[t]{3}{*}{75} & \multirow[t]{3}{*}{ Fusarium sp. } & Cladonia sp.; Stereocaulon sp. & Petrini et al. (1990) \\
\hline & & Peltigera praetextata; Xanthoparmelia taractica & Girlanda et al. (1997) \\
\hline & & Usnea sp. & Kannagara et al. (2008) \\
\hline
\end{tabular}


Table 1 Continued.

\begin{tabular}{|c|c|c|c|}
\hline & Fungi & Host lichen & Reference \\
\hline & & Parmelia sp. & Padhi \& Tayung (2015) \\
\hline & & Arthonia sp.; Bacidina sp. & Govinda Rajulu et al. (2020) \\
\hline \multirow[t]{2}{*}{76} & \multirow[t]{2}{*}{ F. lateritium Nees } & Cladonia sp.; Stereocaulon sp. & Petrini et al. (1990) \\
\hline & & $\begin{array}{l}\text { Heterodermia incana; Polyblastidium dendriticum; } \\
\text { Ramalina arabum; Teloschistes flavicans; Usnea } \\
\text { stigmatoides }\end{array}$ & Vinayaka et al. (2016) \\
\hline \multirow[t]{2}{*}{77} & \multirow{2}{*}{$\begin{array}{l}\text { F. lolii }(\mathrm{Wm} . \mathrm{G} . \mathrm{Sm} .) \text { Sacc. } \\
\text { (= Fusarium heterosporum } \\
\text { Nees ex Fr. })\end{array}$} & Cladonia sp.; Stereocaulon sp. & Petrini et al. (1990) \\
\hline & & $\begin{array}{l}\text { Cladonia fruticulosa; Parmotrema cristiferum; } \\
\text { Ramalina pacifica; Usnea galbinifera }\end{array}$ & Vinayaka et al. (2016) \\
\hline 78 & $\begin{array}{l}\text { F. sublunatum var. } \\
\text { sublunatum Nierenb. }\end{array}$ & Cladonia sp.; Stereocaulon sp. & Petrini et al. (1990) \\
\hline 79 & Geopyxis majalis (Fr.) Sacc. & Pseudevernia intensa & Wijeratne et al. (2012) \\
\hline \multirow[t]{2}{*}{80} & \multirow[t]{2}{*}{ Geotrichum sp. } & Cladonia sp.; Stereocaulon sp. & Petrini et al. (1990) \\
\hline & & $\begin{array}{l}\text { Canoparmelia texana; Heterodermia diademata; } \\
\text { Lobaria kurokawae; Parmotrema tinctorum }\end{array}$ & $\begin{array}{l}\text { Tripathi et al. (2014), } \\
\text { Suryanarayanan et al. (2017) }\end{array}$ \\
\hline \multirow[t]{2}{*}{81} & \multirow{2}{*}{$\begin{array}{l}\text { Gilmaniella humicola G.L. } \\
\text { Barron }\end{array}$} & Bulbothrix meizospora; Parmotrema nilgherrense & Suryanarayanan et al. (2005) \\
\hline & & $\begin{array}{l}\text { Bulbothrix meizospora; Leptogium burnetiae; } \\
\text { Parmotrema nilgherrense; } \text { P. graynum; Punctelia } \\
\text { subrudecta }\end{array}$ & Tripathi et al. (2014) \\
\hline 82 & $\begin{array}{l}\text { Heterobasidion annosum Fr. } \\
\text { Bref. (= Spiniger } \\
\text { meineckellus (Olson) } \\
\text { Stalpers) }\end{array}$ & Cladonia sp.; Stereocaulon sp. & Petrini et al. (1990) \\
\hline 83 & Heteroconium sp. & Cladonia sp.; Stereocaulon sp. & Petrini et al. (1990) \\
\hline 84 & Humicola sp. & $\begin{array}{l}\text { Heterodermia diademata; } H . \text { podocarpa; Lobaria } \\
\text { kurokawae; Parmotrema hababianum; } \\
\text { P. tinctorum; Punctelia rudecta }\end{array}$ & $\begin{array}{l}\text { Tripathi et al. (2014), } \\
\text { Suryanarayanan et al. (2017) }\end{array}$ \\
\hline \multirow[t]{4}{*}{85} & \multirow{4}{*}{$\begin{array}{l}\text { Hypoxylon } \mathrm{sp} . \\
(=\text { Nodulisporium } \mathrm{sp} .)\end{array}$} & Cladonia fruticulosa; Parmotrema reticulatum & Vinayaka et al. (2016) \\
\hline & & $\begin{array}{l}\text { Dermatocarpon miniatum; Melanelia sorediata; } \\
\text { Punctelia borreri }\end{array}$ & Li et al. (2007) \\
\hline & & Hypotrachyna sp. (= Everniastrum sp.) & Zhao et al. (2015) \\
\hline & & $\begin{array}{l}\text { Arthonia } \mathrm{sp} . ; \text { Bacidina } \mathrm{sp} . ; \text { Evernia prunastri; } \\
\text { Hypotrachyna crenata; Parmotrema tinctorum; } \\
\text { Teloschistes flavicans }\end{array}$ & Govinda Rajulu et al. (2020) \\
\hline 86 & $\begin{array}{l}\text { H. anthochroum Berk. \& } \\
\text { Broome }\end{array}$ & $\begin{array}{l}\text { Dirinaria picta; Opegrapha medusulina; Porina } \\
\text { tetracerae }\end{array}$ & Maduranga et al. (2018) \\
\hline 87 & H. fuscum (Pers.) Fr. & Usnea sp. & Basnet et al. (2019a) \\
\hline 88 & $\begin{array}{l}\text { H. fragiforme (Pers.) J. } \\
\text { Kickx f. }\end{array}$ & Lobaria discolor; L. japonica & Oh et al. (2020) \\
\hline 89 & $\begin{array}{l}\text { H. investiens (Schwein.) } \\
\text { M.A. Curtis }\end{array}$ & $\begin{array}{l}\text { Dirinaria consimilis; Evernia prunastri; } \\
\text { Hypotrachyna crenata; Parmotrema } \mathrm{sp} . \\
\text { P. tinctorum; Ramalina pacifica }\end{array}$ & Govinda Rajulu et al. (2020) \\
\hline 90 & $\begin{array}{l}\text { Lasiodiplodia crassispora } \\
\text { T.I Burgess \& P.A. Barber }\end{array}$ & Roccella montagnei & Maduranga et al. (2018) \\
\hline \multirow[t]{3}{*}{91} & \multirow{3}{*}{$\begin{array}{l}\text { L. theobromae (Pat.) Griffon } \\
\text { \& Maubl. }\end{array}$} & Roccella montagnei & Suryanarayanan et al. (2005) \\
\hline & & $\begin{array}{l}\text { Arthonia antillarum; Opegrapha arabica; Pyrenula } \\
\text { ochraceoflava; } P \text {. parvinuclea; Roccella montagnei }\end{array}$ & Maduranga et al. (2018) \\
\hline & & Bacidina sp.; Hypotrachyna crenata & Govinda Rajulu et al. (2020) \\
\hline 92 & $\begin{array}{l}\text { L. pseudotheobromae A.J.L. } \\
\text { Phillips, A. Alves \& Crous }\end{array}$ & Roccella montagnei; Unidentified & Maduranga et al. (2018) \\
\hline 93 & Monilinia sp. (= Monilia sp.) & Heterodermia diademata; Lobaria kurokawae & $\begin{array}{l}\text { Tripathi et al. (2014), } \\
\text { Suryanarayanan et al. (2017) }\end{array}$ \\
\hline 94 & $\begin{array}{l}\text { Mucor hiemalis Wehmer f. } \\
\text { hiemalis }\end{array}$ & Peltigera praetextata; Xanthoparmelia taractica & Girlanda et al. (1997) \\
\hline 95 & M. racemosus Fresen. & Parmotrema reticulatum & $\begin{array}{l}\text { Suryanarayanan et al. (2005), } \\
\text { Tripathi et al. (2014) }\end{array}$ \\
\hline 96 & $\begin{array}{l}\text { Myrothecium inundatum } \\
\text { Tode }\end{array}$ & Ramalina sp. & Basnet et al. (2019b) \\
\hline
\end{tabular}


Table 1 Continued.

\begin{tabular}{|c|c|c|c|}
\hline & Fungi & Host lichen & Reference \\
\hline 97 & Myxotrichum sp. & Cetraria islandica & Yuan et al. (2013) \\
\hline 98 & $\begin{array}{l}\text { Nemania bipapillata (Berk. } \\
\text { \& M.A. Curtis) }\end{array}$ & $\begin{array}{l}\text { Bacidina } \mathrm{sp} . ; \text { Dirinaria consimilis; Hypotrachyna } \\
\text { crenata; Parmotrema } \mathrm{sp} . ; \text { P. tinctorum; } \\
\text { Teloschistes flavicans; Usnea } \mathrm{sp} .\end{array}$ & Govinda Rajulu et al. (2020) \\
\hline 99 & $\begin{array}{l}\text { N. serpens (Pers.) Gray } \\
\text { (= Geniculosporium serpens } \\
\text { Chesters \& Greenh.) }\end{array}$ & Dermatocarpon miniatum & Li et al. (2007) \\
\hline \multirow[t]{5}{*}{100} & \multirow{5}{*}{$\begin{array}{l}\text { Neocosmospora solani } \\
\text { (Mart.) L. Lombard \& Crous } \\
\text { (= Fusarium solani (Mart.) } \\
\text { Sacc.) }\end{array}$} & Xanthoparmelia taractica; Peltigera praetextata & Girlanda et al. (1997) \\
\hline & & $\begin{array}{l}\text { Bulbothrix meizospora; Heterodermia flabellata; } \\
\text { H. hypochraea; Parmotrema crinitum; } \text { P. graynum; } \\
\text { P. praesorediosum; } \text { P. nilgherrense; } \\
\text { P. reticulatum; } P . \text { thomsonii; } \text { Physcia dilatata }\end{array}$ & Suryanarayanan et al. (2005) \\
\hline & & $\begin{array}{l}\text { Heterodermia flabellate; Parmotrema crinitum; } \\
\text { P. graynum; P. thomsonii; Physcia dilatata }\end{array}$ & Li et al. (2007) \\
\hline & & $\begin{array}{l}\text { Bulbothrix meizospora; Flavoparmelia caperata; } \\
\text { Heterodermia flabellata; H. hypochraea; } \\
\text { Leptogium burnetiae; Parmotrema crinitum; } \\
\text { P. graynum; P. praesorediosum; P. nilgherrense; } \\
\text { P. reticulatum; P. thomsonii; Physcia dilatata; } \\
\text { Punctelia subruducta; Usnea sp. }\end{array}$ & Tripathi et al. (2014) \\
\hline & & $\begin{array}{l}\text { Parmotrema cristiferum; } P \text {. reticulatum; Ramalina } \\
\text { pacifica; Usnea stigmatoides }\end{array}$ & Vinayaka et al. (2016) \\
\hline 101 & $\begin{array}{l}\text { Neonectria ditissima (Tul. \& } \\
\text { C. Tul.) Samuels \& Rossman } \\
\text { (= Cylindrocarpon } \\
\text { heteronema (Berk. \& Br.) } \\
\text { Wollenw.) }\end{array}$ & Peltigera praetextata; Xanthoparmelia taractica & Girlanda et al. (1997) \\
\hline 102 & Neurospora sp. & Roccella montagnei & Maduranga et al. (2018) \\
\hline 103 & $\begin{array}{l}\text { N. crassa Shear \& B.O. } \\
\text { Dodge }\end{array}$ & Pyrenula parvinuclea; Pyxine cocoes & Maduranga et al. (2018) \\
\hline 104 & N. terricola Goch. \& Backus & Hypotrachyna cirrhata & Zhang et al. (2009) \\
\hline \multirow[t]{2}{*}{105} & \multirow[t]{2}{*}{ Nigrospora $\mathrm{sp}}$. & Usnea sp. & Kannangara et al. (2008) \\
\hline & & Roccella montagnei & Maduranga et al. (2018) \\
\hline 106 & $\begin{array}{l}\text { N. aurantiaca Mei Wang \& } \\
\text { L. Cai }\end{array}$ & Lobaria discolor; L. japonica & Oh et al. (2020) \\
\hline 107 & $\begin{array}{l}\text { N. chinensis Mei Wang \& L. } \\
\text { Cai }\end{array}$ & Lobaria discolor; L. japonica & Oh et al. (2020) \\
\hline \multirow[t]{3}{*}{108} & \multirow{3}{*}{$\begin{array}{l}\text { N. oryzae (Berk. \& Broome) } \\
\text { Petch }\end{array}$} & Parmotrema reticulatum & Suryanarayanan et al. (2005) \\
\hline & & Leptogium askotense; Parmotrema reticulatum & $\begin{array}{l}\text { Tripathi et al. (2014), } \\
\text { Suryanarayanan et al. (2017) }\end{array}$ \\
\hline & & Parmotrema tinctorum & Govinda Rajulu et al. (2020) \\
\hline \multirow[t]{3}{*}{109} & \multirow[t]{3}{*}{$\begin{array}{l}\text { N. oryzae (Berk. \& Broome) } \\
\text { Petch }(=N . \text { sphaerica } \\
\text { (Sacc.) E.W. Mason) }\end{array}$} & Parmotrema thomsonii & $\begin{array}{l}\text { Suryanarayanan et al. (2005), } \\
\text { Li et al. (2007), Tripathi et al. } \\
(2014)\end{array}$ \\
\hline & & Parmelinella wallichiana & He et al. (2012) \\
\hline & & Opegrapha medusulina; Unidentified & Maduranga et al. (2018) \\
\hline 110 & $\begin{array}{l}\text { Nodulisporium hyalosporum } \\
\text { S.C. Agarwal \& J.K. Misra* }\end{array}$ & Punctelia borreri & Li et al. (2007) \\
\hline 111 & N. sylviforme Deighton* & Melanelia sorediata & Li et al. (2007) \\
\hline 112 & $\begin{array}{l}\text { Ochrocladosporium elatum } \\
\text { (Harz) Crous \& U. Braun (= } \\
\text { C. elatum (Harz.) Nannfeldt) }\end{array}$ & Cladonia sp.; Stereocaulon sp. & Petrini et al. (1990) \\
\hline 113 & $\begin{array}{l}\text { Oidiodendron cf. } \\
\text { echinulatum Barron }\end{array}$ & Cladonia sp.; Stereocaulon sp. & Petrini et al. (1990) \\
\hline 114 & O. flavum Svilv. & Cladonia sp.; Stereocaulon sp. & Petrini et al. (1990) \\
\hline \multirow[t]{2}{*}{115} & \multirow{2}{*}{$\begin{array}{l}\text { Ophiosphaerella korrae } \\
\text { (J. Walker \& A.M. Sm. Bis) } \\
\text { Shoemaker \& C.E. Babc. }\end{array}$} & Unknown & Li et al. (2018) \\
\hline & & Physcia sp. & Li et al. (2019) \\
\hline
\end{tabular}


Table 1 Continued.

\begin{tabular}{|c|c|c|c|}
\hline & Fungi & Host lichen & Reference \\
\hline \multirow[t]{2}{*}{116} & \multirow{2}{*}{ Paecilomyces sp. } & Roccella montagnei & Suryanarayanan et al. (2005) \\
\hline & & $\begin{array}{l}\text { Dirinaria consimilis; Evernia prunastri; } \\
\text { Hypotrachyna crenata; Parmotrema } \mathrm{sp} \text {; } \\
\text { P. tinctorum; Ramalina pacifica; } \text { Teloschistes } \\
\text { flavicans }\end{array}$ & Govinda Rajulu et al. (2020) \\
\hline 117 & Papulospora sp. & $\begin{array}{l}\text { Heterodermia hypochraea; Parmotrema } \\
\text { reticulatum }\end{array}$ & $\begin{array}{l}\text { Suryanarayanan et al. (2005), } \\
\text { Tripathi et al. (2014) }\end{array}$ \\
\hline 118 & $\begin{array}{l}\text { Paraboeremia putaminum } \\
\text { (Speg.) Qian Chen \& L. Cai } \\
\text { (= P. putaminum Speg.) }\end{array}$ & Peltigera praetextata; Xanthoparmelia taractica & Girlanda et al. (1997) \\
\hline 119 & $\begin{array}{l}\text { Paraphaeosphaeria michotii } \\
\text { (Westend) Eriksson }\end{array}$ & Cladonia sp.; Stereocaulon sp. & Petrini et al. (1990) \\
\hline \multirow[t]{7}{*}{120} & \multirow{7}{*}{$\begin{array}{l}\text { Penicillium } \mathrm{sp} .(= \\
\text { Torulomyces } \mathrm{sp} .)\end{array}$} & Cladonia sp.; Stereocaulon sp. & Petrini et al. (1990) \\
\hline & & $\begin{array}{l}\text { Bulbothrix meizospora; Parmotrema reticulatum; } \\
\text { Roccella montagnei }\end{array}$ & Suryanarayanan et al. (2005) \\
\hline & & $\begin{array}{l}\text { Leptogium askotense; Lobaria kurokawae; } \\
\text { Ramalina conduplicans; Usnea sp. }\end{array}$ & Suryanarayanan et al. (2017) \\
\hline & & Pseudocyphellaria $\mathrm{sp}$. & Kannangara et al. (2008) \\
\hline & & $\begin{array}{l}\text { Bulbothrix meizospora; Canoparmelia texana; } \\
\text { Heterodermia podocarpa; Leptogium askotense; } \\
\text { Lobaria kurokawae; Parmotrema hababianum; } \\
\text { P. reticulatum; Phaeophyscia hispidula; Punctelia } \\
\text { rudecta }\end{array}$ & Tripathi et al. (2014) \\
\hline & & Parmelia sp. & Padhi \& Tayung (2015) \\
\hline & & $\begin{array}{l}\text { Arthonia sp.; Bacidina sp.; Hypotrachyna crenata; } \\
\text { Parmotrema tinctorum }\end{array}$ & Govinda Rajulu et al. (2020) \\
\hline 121 & $\begin{array}{l}P . \text { aurantiogriseum } \text { Dierckx } \\
\text { (= } P \text {. viridicatum } \text { Westling) }\end{array}$ & Xanthoparmelia taractica; Peltigera praetextata & Girlanda et al. (1997) \\
\hline 122 & $\begin{array}{l}P . \text { canescens } \text { Sopp }(=P . \\
\text { raciborskii Zaleski })\end{array}$ & Xanthoparmelia taractica; Peltigera praetextata & Girlanda et al. (1997) \\
\hline 123 & P. chrysogenum Thom & $\begin{array}{l}\text { Parmotrema reticulatum; Polyblastidium } \\
\text { dendriticum; Usnea stigmatoides }\end{array}$ & Vinayaka et al. (2016) \\
\hline 124 & P. citrinum Thom & Parmotrema sp. & Samanthi et al. (2015b) \\
\hline 125 & P. griseofulvum Dierckx & Xanthoparmelia taractica; Peltigera praetextata & Girlanda et al. (1997) \\
\hline 126 & P. melinii Thorn & Xanthoparmelia taractica; Peltigera praetextata & Girlanda et al. (1997) \\
\hline 127 & P. purpurogenum Stoll & Xanthoparmelia taractica; Peltigera praetextata & Girlanda et al. (1997) \\
\hline 128 & $\begin{array}{l}\text { P. glaucoalbidum (Desm.) } \\
\text { Houbraken \& Samson }(= \\
\text { Thysanophora penicillioides } \\
\text { (Roum.) Kendrick) }\end{array}$ & Cladonia sp.; Stereocaulon sp. & Petrini et al. (1990) \\
\hline \multirow[t]{3}{*}{129} & \multirow[t]{3}{*}{ Periconia sp. } & Parmotrema sp. & Kannangara et al. (2008) \\
\hline & & $\begin{array}{l}\text { Heterodermia podocarpa; Leptogium askotense; } \\
\text { Lobaria kurokawae; Parmotrema hababianum; } \\
\text { P. tinctorum; Phaeophyscia hispidula; Punctelia } \\
\text { rudecta }\end{array}$ & $\begin{array}{l}\text { Tripathi et al. (2014), } \\
\text { Suryanarayanan et al. (2017) }\end{array}$ \\
\hline & & Parmelia sp. & Wu et al. (2015) \\
\hline \multirow[t]{4}{*}{130} & \multirow[t]{4}{*}{ Pestalotiopsis sp. } & Heterodermia flabellata; Parmotrema thomsonii & $\begin{array}{l}\text { Suryanarayanan et al. (2005), } \\
\text { Li et al. (2007), Tripathi et al. } \\
(2014)\end{array}$ \\
\hline & & Multiclavula sp. & Ding et al. (2009) \\
\hline & & Cetraria islandica & Yuan et al. (2017) \\
\hline & & Bacidina sp. & Govinda Rajulu et al. (2020) \\
\hline 131 & $\begin{array}{l}\text { P. hypodermia }(\mathrm{Niess}) \\
\text { Steyaert }\end{array}$ & $\begin{array}{l}\text { Parmotrema reticulatum; } P . \text { tinctorum; } \\
\text { Teloschistes flavicans }\end{array}$ & Vinayaka et al. (2016) \\
\hline 132 & $\begin{array}{l}\text { P. maculans (Corda) Nag } \\
\text { Raj }\end{array}$ & Parmotrema reticulatum & $\begin{array}{l}\text { Suryanarayanan et al. (2005), } \\
\text { Tripathi et al. (2014) }\end{array}$ \\
\hline 133 & $\begin{array}{l}\text { Pezicula }(=\text { Cryptosporiopsis } \\
\text { sp. })\end{array}$ & Cladonia sp.; Stereocaulon sp. & Petrini et al. (1990) \\
\hline
\end{tabular}


Table 1 Continued.

\begin{tabular}{|c|c|c|c|}
\hline & Fungi & Host lichen & \begin{tabular}{r|} 
Reference \\
\end{tabular} \\
\hline 134 & Phaeoramularia sp. & Cladonia sp.; Stereocaulon sp. & Petrini et al. (1990) \\
\hline 135 & Phaeosphaeria sp. & Heterodermia obscurata & Li et al. (2012) \\
\hline 136 & $\begin{array}{l}\text { Phialocephala fortinii C.J.K. } \\
\text { Wang \& H.E. Wilcox }\end{array}$ & Unknown & Xie et al. (2016) \\
\hline \multirow[t]{5}{*}{137} & \multirow[t]{5}{*}{ Phialophora sp. } & Roccella montagnei & Suryanarayanan et al. (2005) \\
\hline & & Dermatocarpon miniatum & Li et al. (2007) \\
\hline & & Cetrelia braunsiana & He et al. (2012) \\
\hline & & Cladonia ochrochlora & Ye et al. (2013) \\
\hline & & Parmotrema tinctorum; Teloschistes flavicans & Govinda Rajulu et al. (2020) \\
\hline 138 & $\begin{array}{l}\text { P. asteris (Dowson) Burge \& } \\
\text { I. Isaac (= P. fastigiata } \\
\text { (Lagerb. et al) Conant) }\end{array}$ & Cladonia sp.; Stereocaulon sp. & Petrini et al. (1990) \\
\hline 139 & P. verrucosa Medlar & $\begin{array}{l}\text { Heterodermia incana; Ramalina pacifica; Usnea } \\
\text { galbinifera }\end{array}$ & Vinayaka et al. (2016) \\
\hline \multirow[t]{7}{*}{140} & \multirow[t]{7}{*}{ Phoma sp. } & Cladonia sp.; Stereocaulon sp. & Petrini et al. (1990) \\
\hline & & Peltigera praetextata; Xanthoparmelia taractica & Girlanda et al. (1997) \\
\hline & & Punctelia borreri & Li et al. (2007) \\
\hline & & Pseudocyphellaria sp. & Kannangara et al. (2008) \\
\hline & & $\begin{array}{l}\text { Heterodermia incana; Parmotrema tinctorum; } \\
\text { Teloschistes flavicans }\end{array}$ & Vinayaka et al. (2016) \\
\hline & & Unknown & Kim et al. (2018a) \\
\hline & & Arthonia sp. & Govinda Rajulu et al. (2020) \\
\hline 141 & $\begin{array}{l}\text { P. leveillei Boerema \& G.J. } \\
\text { Bollen }\end{array}$ & $\begin{array}{l}\text { Cladonia fruticulosa; Parmotrema reticulatum; } \\
\text { Polyblastidium dendriticum; Ramalina arabum }\end{array}$ & Vinayaka et al. (2016) \\
\hline 142 & $\begin{array}{l}\text { Plagiostoma }(=\text { Diplodina } \\
\text { sp. })\end{array}$ & Cladonia sp.; Stereocaulon sp. & Petrini et al. (1990) \\
\hline 143 & Preussia sp. & Opegrapha medusulina & Maduranga et al. (2018) \\
\hline 144 & $\begin{array}{l}\text { Preussia africana Arenal, } \\
\text { Platas \& Pelàez }\end{array}$ & Ramalina calicaris & Zhang et al. (2012) \\
\hline 145 & $\begin{array}{l}\text { P. tenerifae (Arx \& Guarro) } \\
\text { Kruys }\end{array}$ & Arthonia parantillarum & Maduranga et al. (2018) \\
\hline 146 & Pseudeurotium sp. & \#Not specified - list of several lichens given & Zhang et al. (2016) \\
\hline \multirow[t]{2}{*}{147} & \multirow{2}{*}{$\begin{array}{l}\text { Pseudogymnoascus (Link) } \\
\text { Minnis \& D.L. Lindner (= } \\
\text { Geomyces pannorum (Link) } \\
\text { Sigler et Carmichael }\end{array}$} & Cladonia sp.; Stereocaulon sp. & Petrini et al. (1990) \\
\hline & & Xanthoparmelia taractica; Peltigera praetextata & Girlanda et al. (1997) \\
\hline 148 & $\begin{array}{l}\text { Purpureocillium lilacinum } \\
\text { (Thom) Luangsa-ard, } \\
\text { Houbraken, Hywel-Jones \& } \\
\text { Samson (= Paecilomyces } \\
\text { lilacinus (Thorn) Samson) }\end{array}$ & Xanthoparmelia taractica; Peltigera praetextata & Girlanda et al. (1997) \\
\hline 149 & P. variotii Bainier & Cladonia sp.; Stereocaulon sp. & Petrini et al. (1990) \\
\hline 150 & $\begin{array}{l}\text { Ramichloridium cf. } \\
\text { apiculatum (Miller et al.) De } \\
\text { Hoog }\end{array}$ & Cladonia sp.; Stereocaulon sp. & Petrini et al. (1990) \\
\hline 151 & Ramularia sp. & Cladonia sp.; Stereocaulon sp. & Petrini et al. (1990) \\
\hline 152 & $\begin{array}{l}\text { Rigidoporus vinctus (Berk.) } \\
\text { Ryvarden }\end{array}$ & Roccella montagnei & Maduranga et al. (2018) \\
\hline 153 & Rhizoctonia sp. & Punctelia subruducta & Tripathi et al. (2014) \\
\hline 154 & R. solani J.G. Kühn & $\begin{array}{l}\text { Heterodermia incana; Parmotrema cristiferum; } \\
\text { Usnea stigmatoides }\end{array}$ & Vinayaka et al. (2016) \\
\hline 155 & Rhizopus sp. & Roccella montagnei & Suryanarayanan et al. (2005) \\
\hline 156 & $\begin{array}{l}\text { Rhizosphaera kalkhoffii } \\
\text { Bubak }\end{array}$ & Cladonia sp.; Stereocaulon sp. & Petrini et al. (1990) \\
\hline 157 & Schizophyllum commune Fr. & Opegrapha medusulina; Unidentified & Maduranga et al. (2018) \\
\hline 158 & Scopulariopsis sp. & Cladonia sp.; Stereocaulon sp. & Petrini et al. (1990) \\
\hline
\end{tabular}


Table 1 Continued.

\begin{tabular}{|c|c|c|c|}
\hline & Fungi & \begin{tabular}{|l|} 
Host lichen \\
\end{tabular} & $\begin{array}{r}\text { Reference } \\
\end{array}$ \\
\hline & & $\begin{array}{l}\text { Cladonia coniocraea; Dermatocarpon miniatum; } \\
\text { Melanelia sorediata; Parmelia } \mathrm{sp} . ; \text { Punctelia } \\
\text { borreri; Zeroviella mandschurica }\end{array}$ & Li et al. (2007) \\
\hline 159 & $\begin{array}{l}\text { Scutula } \mathrm{sp} . \\
(=\text { Karsteniomyces } \mathrm{sp} .)\end{array}$ & Xanthoparmelia taractica; Peltigera praetextata & Girlanda et al. (1997) \\
\hline \multirow[t]{2}{*}{160} & \multirow[t]{2}{*}{ Sordaria sp. } & $\begin{array}{l}\text { Heterodermia diademata; } \text { H. podocarpa; } \\
\text { Leptogium askotense; Lobaria kurokawae; } \\
\text { Parmotrema tinctorum; Phaeophyscia hispidula; } \\
\text { Punctelia rudecta; Usnea sp. }\end{array}$ & $\begin{array}{l}\text { Tripathi et al. (2014), } \\
\text { Suryanarayanan et al. (2017) }\end{array}$ \\
\hline & & Unidentified & Maduranga et al. (2018) \\
\hline \multirow[t]{2}{*}{161} & \multirow[t]{2}{*}{$\begin{array}{l}\text { S. fimicola (Roberge ex } \\
\text { Desm.) Ces. \& De Not. }\end{array}$} & Parmotrema reticulatum & $\begin{array}{l}\text { Tripathi et al. (2014), } \\
\text { Suryanarayanan et al. (2005) }\end{array}$ \\
\hline & & $\begin{array}{l}\text { Myelochroa sp.; Parmotrema sp.; Phaeophyscia } \\
\text { sp.; Physcia sp.; Stereocaulon sp. }\end{array}$ & Oh et al. (2020) \\
\hline 162 & $\begin{array}{l}\text { Spegazzinia tessarthra } \\
\text { (Berk. \& M.A. Curtis) Sacc. }\end{array}$ & Heterodermia flabellata & $\begin{array}{l}\text { Suryanarayanan et al. (2005), } \\
\text { Li et al. (2007), Tripathi et al. } \\
(2014)\end{array}$ \\
\hline 163 & S. lobulata Thrower & Punctelia rudecta & $\begin{array}{l}\text { Tripathi et al. (2014), } \\
\text { Suryanarayanan et al. (2017) }\end{array}$ \\
\hline 164 & Spiniger sp. & Cladonia sp.; Stereocaulon sp. & Petrini et al. (1990) \\
\hline 165 & Sporormiella sp. & Zeroviella mandschurica & Li et al. (2007) \\
\hline \multirow[t]{2}{*}{166} & \multirow{2}{*}{$\begin{array}{l}\text { S. intermedia (Auersw.) } \\
\text { Ahmed \& Cain }\end{array}$} & Roccella montagnei & Suryanarayanan et al. (2005) \\
\hline & & $\begin{array}{l}\text { Arthonia } \text { sp.; Dirinaria consimilis; Hypotrachyna } \\
\text { crenata; Parmotrema } \mathrm{sp} . ; \text { P. tinctorum; Ramalina } \\
\text { pacifica; Teloschistes flavicans }\end{array}$ & Govinda Rajulu et al. (2020) \\
\hline \multirow[t]{2}{*}{167} & \multirow{2}{*}{$\begin{array}{l}\text { S. minima (Auersw.) S.I. } \\
\text { Ahmed \& Cain }\end{array}$} & Zeroviella mandschurica & Li et al. (2007) \\
\hline & & $\begin{array}{l}\text { Canoparmelia texana; Heterodermia diademata; } \\
\text { H. podocarpa; Lobaria kurokawae; Parmotrema } \\
\text { hababianum; P. tinctorum; Phaeophyscia } \\
\text { hispidula; Punctelia rudecta; Ramalina } \\
\text { conduplicans; Usnea } \text { sp. }\end{array}$ & $\begin{array}{l}\text { Tripathi et al. (2014), } \\
\text { Suryanarayanan et al. (2017) }\end{array}$ \\
\hline 168 & $\begin{array}{l}\text { S. muskokensis (Cain) S.I. } \\
\text { Ahmed \& Cain }\end{array}$ & Zeroviella mandschurica & Li et al. (2007) \\
\hline 169 & Sporothrix sp. & $\begin{array}{l}\text { Dermatocarpon miniatum; Parmelia sp.; Ramalina } \\
\text { sinensis }\end{array}$ & Li et al. (2007) \\
\hline 170 & $\begin{array}{l}\text { Sydowia polyspora (Bref. \& } \\
\text { Tavel) E. Müll. (= } \\
\text { Hormonema dematioides } \\
\text { Lagerberg et Melin) }\end{array}$ & Cladonia sp.; Stereocaulon sp. & Petrini et al. (1990) \\
\hline 171 & Taeniolella sp. & Teloschistes flavicans & Govinda Rajulu et al. (2020) \\
\hline \multirow[t]{5}{*}{172} & \multirow[t]{5}{*}{ Talaromyces $\mathrm{sp}$. } & Leptogium askotense; Lobaria kurokawae & Tripathi et al. (2014) \\
\hline & & $\begin{array}{l}\text { Canoparmelia texana; Heterodermia podocarpa; } \\
\text { Leptogium askotense; Lobaria kurokawae; } \\
\text { Parmotrema hababianum; Phaeophyscia hispidula; } \\
\text { Punctelia rudecta }\end{array}$ & Suryanarayanan et al. (2017) \\
\hline & & Porina tetracerae & Maduranga et al. (2018) \\
\hline & & \begin{tabular}{|l|} 
Xanthoparmelia angustiphylla \\
\end{tabular} & Yuan et al. (2018) \\
\hline & & Parmotrema tinctorum; Ramalina pacifica & Govinda Rajulu et al. (2020) \\
\hline 173 & $\begin{array}{l}\text { T. funiculosus (Thom.) } \\
\text { Samson }\end{array}$ & Diorygma hieroglyphicum & Padhi et al. (2019) \\
\hline 174 & $\begin{array}{l}\text { T. tratensis Manoch, } \\
\text { Dethoup \& N. Yilmaz } \\
\end{array}$ & Lecanora sp. & Jayakumar et al. (2016) \\
\hline 175 & $\begin{array}{l}\text { T. verruculosus (Peryronel) } \\
\text { Samson, N. Yilmaz, Frisvad } \\
\text { \& Seifert (= Penicillium } \\
\text { verruculosum Peyronel) }\end{array}$ & Peltigera praetextata; Xanthoparmelia taractica & Girlanda et al. (1997) \\
\hline
\end{tabular}


Table 1 Continued.

\begin{tabular}{|c|c|c|c|}
\hline & Fungi & Host lichen & Reference \\
\hline 176 & $\begin{array}{l}\text { Teichospora striata } \text { (Kohlm. } \\
\text { \& Volkm. -Kohlm.) Jaklitsch } \\
\text { \& Voglmayr (= Floricola } \\
\text { striata Kohlm. \& Volkm- } \\
\text { Kohlm.) }\end{array}$ & Umbilicaria sp. & Li et al. (2016) \\
\hline \multirow[t]{2}{*}{177} & \multirow[t]{2}{*}{ Thielavia sp. } & $\begin{array}{l}\text { Melanelia sorediata; Parmelia } \text { sp.; Punctelia } \\
\text { borreri }\end{array}$ & Li et al. (2007) \\
\hline & & Dermatocarpon miniatum; Melanelia sorediata & Vinayaka et al. (2016) \\
\hline \multirow[t]{3}{*}{178} & \multirow{3}{*}{$\begin{array}{l}\text { Tolypocladium } \mathrm{sp} . \\
(=\text { Chaunopycnis } \mathrm{sp} .)\end{array}$} & Peltigera praetextata; Xanthoparmelia taractica & Girlanda et al. (1997) \\
\hline & & Parmelia sp. & Hu et al. (2017) \\
\hline & & Cladonia sp.; Stereocaulon sp. & Petrini et al. (1990) \\
\hline 179 & T. cylindrosporum W. Gams & Lethariella zahlbruckneri & Li et al. (2015b) \\
\hline 180 & T. geodes W. Gams & Peltigera praetextata; Xanthoparmelia taractica & Girlanda et al. (1997) \\
\hline 181 & $\begin{array}{l}\text { T. inflatum W. Gams (= T. } \\
\text { niveum (Rostr.) Bissett) }\end{array}$ & Peltigera praetextata; Xanthoparmelia taractica & Girlanda et al. (1997) \\
\hline 182 & Torula sp. & Cladonia sp.; Stereocaulon sp. & Petrini et al. (1990) \\
\hline 183 & Tremella sp. & \#Not specified - list of several lichens given & Zhang et al. (2016) \\
\hline 184 & Trichobotrys sp. & & Li et al. (2007) \\
\hline \multirow[t]{4}{*}{185} & \multirow[t]{4}{*}{ Trichoderma sp. } & Roccella montagnei & Suryanarayanan et al. (2005) \\
\hline & & $\begin{array}{l}\text { Cladonia coniocraea; Melanelia sorediata; } \\
\text { Punctelia borreri }\end{array}$ & Li et al. (2007) \\
\hline & & Phaeophyscia hispidula; Punctelia rudecta & Tripathi et al. (2014) \\
\hline & & Pamelia sp. & Padhi \& Tayung (2015) \\
\hline 186 & T. hamatum (Bon.) Bain. & Cladonia sp.; Stereocaulon sp. & Petrini et al. (1990) \\
\hline \multirow[t]{5}{*}{187} & \multirow[t]{5}{*}{ T. harzianum Rifai } & $\begin{array}{l}\text { Cladonia sp.; Stereocaulon sp.; Xanthoparmelia } \\
\text { taractica }\end{array}$ & Petrini et al. (1990) \\
\hline & & Peltigera praetextata & Girlanda et al. (1997) \\
\hline & & $\begin{array}{l}\text { Heterodermia flabellata; Parmotrema crinitum; } \\
\text { P. thomsonii; Physcia dilatata }\end{array}$ & Suryanarayanan et al. (2005) \\
\hline & & $\begin{array}{l}\text { Heterodermia flabellata; Parmotrema crinitum; } \\
\text { P. thomsonii; Physcia dilatata }\end{array}$ & Li et al. (2007) \\
\hline & & $\begin{array}{l}\text { Heterodermia flabellata; Parmotrema crinitum; } \\
\text { P. thomsonii; Physcia dilatata; Usnea } \mathrm{sp.}\end{array}$ & Tripathi et al. (2014) \\
\hline 188 & T. koningii Oud. & Cladonia sp.; Stereocaulon sp. & Petrini et al. (1990) \\
\hline 189 & $\begin{array}{l}\text { T. polysporum (Link ex } \\
\text { Pers.) Rifai }\end{array}$ & Peltigera praetextata; Xanthoparmelia taractica & Girlanda et al. (1997) \\
\hline \multirow[t]{3}{*}{190} & \multirow[t]{3}{*}{ T. viride Pers. ex S. F. Gray } & Cladonia sp.; Stereocaulon sp. & Petrini et al. (1990) \\
\hline & & Peltigera praetextata; Xanthoparmelia taractica & Girlanda et al. (1997) \\
\hline & & $\begin{array}{l}\text { Cladonia fruticulosa; Parmotrema tinctorum; } \\
\text { Ramalina arabum; Usnea stigmatoides }\end{array}$ & Vinayaka et al. (2016) \\
\hline 191 & $\begin{array}{l}\text { Trichophyton roseum } \mathrm{E} . \\
\text { Bodin }\end{array}$ & Parmotrema graynum & Tripathi et al. (2014) \\
\hline 192 & $\begin{array}{l}\text { Umbelopsis vinacea (Dixon- } \\
\text { Stew.) Arx (= Mortierella } \\
\text { vinacea } \text { Dixon-Stewart) }\end{array}$ & Cladonia sp.; Stereocaulon sp. & Petrini et al. (1990) \\
\hline 193 & Verticillium sp. & Cladonia sp.; Stereocaulon sp. & Petrini et al. (1990) \\
\hline \multirow[t]{3}{*}{194} & \multirow[t]{3}{*}{ Xylaria sp. } & Leptogium saturninum & Wu et al. (2011) \\
\hline & & $\begin{array}{l}\text { Canoparmelia texana; Heterodermia diademata; } \\
\text { H. podocarpa; Leptogium askotense; Lobaria } \\
\text { kurokawae; Parmotrema hababianum; } \\
\text { P. tinctorum; Phaeophyscia hispidula; Punctelia } \\
\text { rudecta; Ramalina conduplicans; Usnea sp. }\end{array}$ & $\begin{array}{l}\text { Tripathi et al. (2014), } \\
\text { Suryanarayanan et al. (2017) }\end{array}$ \\
\hline & & Opegrapha medusulina & Maduranga et al. (2018) \\
\hline 195 & X. apiculata Cooke & Arthonia sp. & Govinda Rajulu et al. (2020) \\
\hline 196 & $X$. arbuscula Sacc. & Lobaria discolor; L. japonica & Oh et al. (2020) \\
\hline 197 & X. castorea Berk. & Roccella montagnei & Maduranga et al. (2018) \\
\hline 198 & X. feejeensis (Berk.) Fr. & Opegrapha medusulina & Maduranga et al. (2018) \\
\hline 199 & X. grammica (Mont.) Mont. & Menegazzia sp. & Kim et al. (2018b) \\
\hline
\end{tabular}


Table 1 Continued.

\begin{tabular}{|c|c|c|c|}
\hline & Fungi & Host lichen & Reference \\
\hline 200 & X. hypoxylon (L.) Grev. & Parmotrema reticulatum & $\begin{array}{l}\text { Suryanarayanan et al. (2005), } \\
\text { Tripathi et al. (2014) }\end{array}$ \\
\hline 201 & $\begin{array}{l}\text { X. primorskensis Y.M. Ju, } \\
\text { H.M. Hsieh, Lar. N. } \\
\text { Vassiljeva \& Akulov }\end{array}$ & $\begin{array}{l}\text { Hypotrachyna crenata; Parmotrema sp.; Ramalina } \\
\text { pacifica; Usnea } \mathrm{sp} .\end{array}$ & Govinda Rajulu et al. (2020) \\
\hline 202 & $\begin{array}{l}\text { X. psidii J.D. Rogers \& } \\
\text { Hemmes }\end{array}$ & Amandinea sp. & Maduranga et al. (2018) \\
\hline 203 & X. vaporaria Berk. & $\begin{array}{l}\text { Parmotrema cristiferum; Ramalina arabum; } \\
\text { R. pacifica }\end{array}$ & Vinayaka et al. (2016) \\
\hline 204 & Zygosporium sp. & Parmotrema tinctorum & Govinda Rajulu et al. (2020) \\
\hline
\end{tabular}

Note: \#List of 17 species utilized by Zhang et al. (2016) for isolation of ELF. Acarospora molybdina (Ach.) Trevis., Allantoparmelia alpicola (Th. Fr.) Essl., Cetraria delisei (Bory ex Schaer.) Nyl. (= Cetrariella delisei (Bory ex Schaer.) Kärnefelt \& A. Thell), Cladonia arbuscula (Wallr.) Flot., C. borealis S. Stenroos, C. pocillum (Ach.) O.J. Rich., Nephromopsis nivalis (L.) Divakar, A. Crespo \& Lumbsch (= Flavocetraria nivalis (L.) Kärnefelt \& A. Thell), Ochrolechia frigida (Sw.) Lynge, Peltigera canina (L.) Willd., Placynthium asperellum (Ach.) Trevis., Pseudephebe pubescens (L.) M. Choisy, Stereocaulon alpinum Laurer, S. botryosum Ach., S. vesuvianum Pers., Umbilicaria aprina Nyl., U. arctica (Ach.) Nyl., U. torrefacta (Lightf.) Schrad. *status of the species is unconfirmed under the genus.

The ELFs identified till date belongs to three phyla, 32 orders, 70 families and 112 genera (Table 2, according to Wijayawardene et al. 2020). Majority of them belong to Ascomycota, followed by Basidiomycota, and very few belong to Mucoromycota. Within the Ascomycota, the classes Dothideomycetes and Sordariomycetes represent the largest number of ELFs reported so far. Alternaria, Aspergillus, Chaetomium, Cladosporium, Curvularia, Fusarium, Penicillium, Trichoderma, and Xylaria are some of the most common genera. Alternaria alternata, Aspergillus flavus, A. niger, Neocosmospora solani, and Trichoderma harzianum are the most frequently occurring ELF species.

Table 2 Classification of endolichenic fungi (sensu Wijayawardene et al. 2020)

\begin{tabular}{|c|c|c|c|c|}
\hline Phylum & Class & Order & \begin{tabular}{|c|} 
Family \\
\end{tabular} & $\begin{array}{c}\text { Genus } \\
\end{array}$ \\
\hline Ascomycota & Dothideomycetes & Botryosphaeriales & Botryosphaeriaceae & Endomelanconiopsis; Lasiodiplodia \\
\hline & & Capnodiales & Cladosporiaceae & Cladosporium \\
\hline & & & Dissoconiaceae & Ramichloridium \\
\hline & & & Mycosphaerellaceae & Phaeoramularia; Ramularia \\
\hline & & Dothideales & Dothideaceae & Sydowia \\
\hline & & & Saccotheciaceae & Aureobasidium \\
\hline & & \multicolumn{2}{|c|}{ Dothideales genus incertae sedis } & Rhizosphaera \\
\hline & & \multicolumn{2}{|c|}{$\begin{array}{l}\text { Kirschsteiniotheliales genus incertae } \\
\text { sedis }\end{array}$} & Taeniolella \\
\hline & & Pleosporales & Biatriosporaceae & Biatriospora \\
\hline & & & Coniothyriaceae & Ochrocladosporium \\
\hline & & & Corynesporascaceae & Corynespora \\
\hline & & & Didymellaceae & $\begin{array}{l}\text { Ascochyta } ; \text { Epicoccum; } \\
\text { Paraboeremia } \text { Phoma }\end{array}$ \\
\hline & & & Didymosphaeriaceae & Paraphaeosphaeria; Spegazzinia \\
\hline & & & Periconiaceae & Periconia \\
\hline & & & Pleosporaceae & Alternaria; Curvularia \\
\hline & & & Phaeosphaeriaceae & Ophiosphaerella; Phaeosphaeria \\
\hline & & & Sporormiaceae & Preussia; Sporormiella \\
\hline & & & Teichosporaceae & Teichospora \\
\hline & & & Torulaceae & Torula \\
\hline & \multicolumn{3}{|c|}{ Dothideomycetes genera incertae sedis } & Bahusakala; Heteroconium \\
\hline & Eurotiomycetes & Chaetothyriales & Herpotrichiellaceae & $\begin{array}{l}\text { Cladophialophora; Exophiala; } \\
\text { Phialophora }\end{array}$ \\
\hline & & Eurotiales & Aspergillaceae & Aspergillus; Penicillium \\
\hline & & & Thermoascaceae & Paecilomyces \\
\hline
\end{tabular}


Table 2 Continued.

\begin{tabular}{|c|c|c|c|c|}
\hline Phylum & Class & Order & Family & Genus \\
\hline & & & Trichocomaceae & $\begin{array}{l}\text { Talaromyces; Thysanophora; } \\
\text { Byssochlamys }\end{array}$ \\
\hline & & Onygenales & Arthrodermataceae & Trichophyton \\
\hline & & & Onygenaceae & Chrysosporium \\
\hline & Lecanoromycetes & Lecanorales & Ramalinaceae & Scutula \\
\hline & Leotiomycetes & Chaetomellales & Chaetomellaceae & Chaetomella \\
\hline & & Helotiales & Amorphothecaceae & Myxotrichum \\
\hline & & & Dermateaceae & Pezicula \\
\hline & & & Mollisiaceae & Phialocephala \\
\hline & & & Myxotrichaceae & Oidiodendron \\
\hline & & & Ploettnerulaceae & Cadophora; Cylindrosporium \\
\hline & & & Sclerotiniaceae & Botrytis; Monilinia \\
\hline & & \multicolumn{2}{|c|}{ Helotiales genus incertae sedis } & Cryptocline \\
\hline & & Thelebolales & Pseudeurotiaceae & Pseudeurotium; Pseudogymnoascus \\
\hline & \multicolumn{2}{|c|}{$\begin{array}{l}\text { Leotiomycetes family and genus incertae } \\
\text { sedis }\end{array}$} & $\begin{array}{l}\text { Hamatocanthoscypha } \\
\text { ceae }\end{array}$ & Chalara \\
\hline & Pezizomycetes & Pezizales & Tarzettaceae & Geopyxis \\
\hline & Saccharomycetes & Saccharomycetales & Dipodascaceae & Geotrichum \\
\hline & Sordariomycetes & Amphisphaeriales & Apiosporaceae & Arthrinium; Nigrospora \\
\hline & & & Sporocadaceae & Broomella; Pestalotiopsis \\
\hline & & Coniochaetales & Coniochaetaceae & Coniochaeta \\
\hline & & Diaporthales & Cytosporaceae & Cytospora \\
\hline & & & Diaporthaceae & Diaporthe \\
\hline & & & Gnomoniaceae & Plagiostoma \\
\hline & & Glomerellales & Glomerellaceae & Colletotrichum \\
\hline & & & Plectosphaerellaceae & Verticillium \\
\hline & & Hypocreales & Bionectriaceae & Acremonium; Cylindromonium \\
\hline & & & Cordycipitaceae & Cordyceps \\
\hline & & & Hypocreaceae & Trichoderma \\
\hline & & & Nectriaceae & $\begin{array}{l}\text { Aphanocladium; Calonectria; } \\
\text { Cosmospora; Fusarium; } \\
\text { Neocosmospora; Neonectria }\end{array}$ \\
\hline & & & Ophiocordycipitaceae & Purpureocillium; Tolypocladium \\
\hline & & & Stachybotryaceae & Myrothecium \\
\hline & & Microascales & Ceratocystidaceae & Berkeleyomyces \\
\hline & & & Microascaceae & Scopulariopsis \\
\hline & & Ophiostomatales & Ophiostomataceae & Sporothrix \\
\hline & & Sordariales & Chaetomiaceae & Chaetomium; Humicola; Thielavia \\
\hline & & & Lasiosphaeriaceae & Apiosordaria \\
\hline & & & Sordariaceae & Neurospora; Sordaria \\
\hline & & Xylariales & Graphostromataceae & Biscogniauxia \\
\hline & & & Hypoxylaceae & Daldinia; Hypoxylon \\
\hline & & & Xylariaceae & Nemania; Xylaria \\
\hline & & & Zygosporiaceae & Zygosporium \\
\hline & \multicolumn{3}{|c|}{ Sordariomycetes genus incertae sedis (?) } & Papulospora \\
\hline \multicolumn{4}{|c|}{ Ascomycota genera incertae sedis } & Bispora; Gilmaniella; Trichobotrys \\
\hline \multirow[t]{8}{*}{ Basidiomycota } & Agaricomycetes & Cantharellales & Ceratobasidiaceae & Rhizoctonia \\
\hline & & Agaricales & Schizophyllaceae & Schizophyllum \\
\hline & Bartheletiomycetes & Russulales & Bondarzewiaceae & Heterobasidion; Spiniger \\
\hline & Cystobasidiomycetes & Cystobasidiales & Cystobasidiaceae & Cystobasidium \\
\hline & Tremellomycetes & Tremellales & Tremellaceae & Tremella \\
\hline & & Trichosporonales & Trichosporonaceae & Apiotrichum \\
\hline & & Polyporales & Polyporaceae & Cerrena \\
\hline & & & Meripilaceae & Rigidoporus \\
\hline \multirow[t]{3}{*}{ Mucoromycota } & Mucoromycetes & Mucorales & Mucoraceae & Mucor \\
\hline & & & Rhizopodaceae & Rhizopus \\
\hline & Umbelopsidomycetes & Umbelopsidales & Umbelopsidaceae & Umbelopsis \\
\hline
\end{tabular}




\section{ELF host diversity and ecology}

The ELFs are not host-specific and behave as generalists (Chagnon et al. 2016). Studies have shown that certain endolichenic fungal species are widespread and occur in different lichen species (Tripathi et al. 2014). The ELF communities, their incidence, diversity, and composition reflect the interplay of climatic patterns, geographic separation, host type, and host lineage (U'Ren et al. 2012). Their composition depends on the environment of the lichens and the chemical ambience of the thalli (Arnold \& Herre 2003, Suryanarayanan \& Thennarasan 2004). In addition, the altitude and the geographical location also affect the ELF composition as disclosed by 18S rDNA sequencing study (Wang et al. 2016). The compilation of ELF studies carried out to date shows that a total of 114 species of lichens belonging to 22 families were explored for ELFs (see name list). In few cases, the host species are partially identified (Petrini et al. 1990, Kannangara et al. 2008, Chen et al. 2014, 2019, Samanthi et al. 2015a, Wang et al. 2017, Govinda Rajulu et al. 2020) or unidentified (Wang et al. 2012, Kim et al. 2014, 2018a, b, Li et al. 2015a, Xie et al. 2016, Li et al. 2018). The present study shows that only a fraction of the 20000 species of lichens (Lücking et al. 2017) have been explored for their ELF. A majority of the host lichens studied for their ELF belong to the macro lichens (foliose and fruticose), probably because they are easy to handle and identify. Among them, members of families Parmeliaceae, Physciaceae, Peltigeraceae and Cladoniaceae have been studied more intensely. Also, species of Parmotrema were most preferred for isolation of ELF (Name list. Lichen hosts of endolichenic fungi).

It is clear from the species diversity of ELF that most of the taxa (Alternaria, Aspergillus, Chaetomium, Cladosporium, Curvularia, Fusarium, Penicillium, Trichoderma, and Xylaria) are generalists that occur as saprotrophs (Arnold 2009). Their invasion into lichen thallus and association with the photobiont of the lichen suggests that they form an opportunistic interaction. It is speculated that the high level of fungal diversity may be due to highly porous and heterogeneous nature of the lichen thalli (Stone et al. 2012). Petrini et al. (1990) categorized ELFs into three ecological groups, of which the first one comprised of the species known as soil and litter inhabitants. Several of these belonged to genera frequently identified as plant pathogens or plant symbionts. The other groups reported as epiphytes or pathogens of higher plants (Dettki 1998, Arnold 2003). Several examples can be cited to show ELFs are not specific to lichens. Alternaria alternata isolated from a large number of lichens found in angiosperm Hedychium spicatum Ham ex Sm. (Sarma et al. 2020) and gymnosperm Pinus tabulaeformis Carr. (Guo et al. 2004). Cladosporium cladosporioides is also isolated from the leaves of medicinally important pteridophyte Huperzia serrata (Thunb. ex Murray) Trev. (Zhang et al. 2011).

Name list. Lichen hosts of endolichenic fungi

\section{Acarosporaceae}

Acarospora molybdina (Ach.) Trevis.

\section{Arthoniaceae}

Arthonia sp.

Arthonia antillarum (Fée) Nyl.

Arthonia parantillarum Aptroot

\section{Caliciaceae}

Dirinaria consimilis (Stirt.) D.D. Awasthi

\section{Cladoniaceae}

Cladonia sp.

C. arbuscula (Wallr.) Flot.

C. borealis S. Stenroos

C. grisea (Ahti) Trass

C. coniocraea (Flörke) Spreng.

C. evansii Abbayes

C. fruticulosa Kremp. 
C. ochrochlora Flörke

C. pocillum (Ach.) O.J. Rich.

Stereocaulon $\mathrm{sp}$.

Stereocaulon alpinum Laurer

S. botryosum Ach.

S. vesuvianum Pers.

\section{Collemataceae}

Leptogium askotense D.D. Awasthi

L. burnetiae Dodge

L. saturninum (Dicks.) Nyl.

\section{Graphidaceae}

\section{Hydnaceae}

Diorygma hieroglyphicum (Pers.) Staiger \& Kalb

Multiclavula sp.

\section{Lecanoraceae}

\section{Lobariaceae}

Lobaria discolor (Bory) Hue

Lobaria japonica (Zahlbr.) Asahina

\section{Ochrolechiaceae}

Ochrolechia frigida (Sw.) Lynge

\section{Parmeliaceae}

Allantoparmelia alpicola (Th. Fr.) Essl.

Bulbothrix meizospora (Nyl.) Hale

Canoparmelia texana (Tuck.) Elix \& Hale

Cetraria islandica (L.) Ach.

Cetraria delisei (Bory ex Schaer.) Nyl.

Cetrelia sp.

C. braunsiana (Müll. Arg.) W.L. Culb. \& C.F. Culb.

Evernia prunastri (L) Ach.

Flavoparmelia caperata (L.) Hale

Hypotrachyna cirrhata (Fr.) Divakar et al.

H. crenata (Kurok.) Hale

H. nepalensis (Taylor) Divakar et al.

Lethariella zahlbruckneri (Du Rietz) Krog.

Melanelia sorediata (Ach.) Goward \& Ahti

Menegazzia sp.

Nephromopsis nivalis (L.) Divakar et al.

Parmelia sp.

Parmelinella wallichiana (Taylor) Elix \& Hale

Parmotrema sp.

P. austrosinense (Zahlbr.) Hale

$P$. cristiferum (Taylor) Hale

P. critinum (Ach.) Choisy

$P$. graynum (Hue) Hale

$P$. hababianum (Gyeln.) Hale

$P$. nilgherrense (Nyl.) Hale

$P$. praesorediosum (Ny) Hale

$P$. ravum (Krog Swinscow) Sérus.

$P$. reticulatum (Taylor) Choisy

P. thomsonii (Stirt.) A. Crespo, Divakar \& Elix

P. tinctorum (Despr. ex Nyl.) Hale 
Pseudevernia intensa (Nyl.) Hale \& W.L. Culb.

Pseudephebe pubescens (L.) M. Choisy

Punctelia borreri (Turner) Krog

P. rudecta (Ach.) Krog.

P. subrudecta (Nyl.) Krog

Usnea sp.

$U$. aciculifera Vain.

U. cavernosa Tuck.

U. galbinifera Asahina

U. longissima Ach.

U. stigmatoides $\mathrm{G}$. Awasthi

Xanthoparmelia angustiphylla (Gyelnik) Hale.

$X$. taractica (Kremp.) Hale

\section{Peltigeraceae}

Lobaria kurokawae Yoshim.

L. retigera (Bory) Trevis.

Peltigera canina (L.) Willd.

P. elisabethae var. mauritzii (Gyeln.) J.C. Wei

$P$. praetextata (Flörke ex Sommerf.) Zopf

Pseudocyphellaria sp.

Ricasolia quercizans (Michx.) Stizenb.

\section{Pertusariaceae}

Pertusaria laeviganda $\mathrm{Nyl}$.

\section{Physciaceae}

Amandinea sp.

Dirinaria picta (Sw.) Clem. \& Shear

Heterodermia diademata (Taylor) D.D. Awasthi

H. flabellata (Fée) D.D. Awasthi

H. hypochraea (Vain.) Swinsc \& Krog

H. incana (Stirt.) D.D. Awasthi

H. obscurata (Nyl.) Trevis.

H. podocarpa (Bél) D.D. Awasthi

Phaeophyscia hispidula (Ach.) Moberg

Physcia sp.

$P$. dilatata Nyl.

Polyblastidium dendriticum (Pers.) Kalb

Pyxine cocoes (Sw.) Nyl.

\section{Placynthiaceae}

Placynthium asperellum (Ach.) Trevis.

\section{Porinaceae}

Porina tetracerae (Ach.) Müll. Arg.

\section{Pyrenulaceae}

Pyrenula sp.

P. ochraceoflava (Nyl.) R.C. Harris

P. parvinuclea (Meyen \& Flot.) Aptroot

\section{Ramalinaceae}

Bacidina sp.

Ramalina sp.

$R$. arabum (Dill. ex Ach.) Meyen \& Flot.

R. calicaris (L.) Röhl.

$R$. conduplicans Vain.

R. pacifica Asahina 


\section{Roccellaceae}

Opegrapha arabica (Müll. Arg.) Vain.

O. medusulina $\mathrm{Nyl}$.

Roccella montagnei Bél.

\section{Teloschistaceae}

Teloschistes flavicans (Sw.) Norman

Zeroviella mandschurica (Zahlbr.) S.Y. Kondr. \& Hur

\section{Umbilicariaceae}

Umbilicaria sp.

$U$. aprina $\mathrm{Nyl}$.

$U$. $\operatorname{arctica}$ (Ach.) Nyl.

Verrucariaceae

U. torrefacta (Lightf.) Schrad.

Dermatocarpon miniatum (L.) W. Mann.

\section{Conclusion}

Lichens establish successful symbiotic associations for over 600 million years (Yuan et al. 2005). However, the origin and evolution of ELFs is not well-understood as lichens, and their components do not preserve well as fossils. Some of the fossil lichen thalli obtained from the Lower Devonian period showed evidence of endolichenic fungal association, which suggests that ELF association existed since 415 million years ago. Such a long association of these fungi with lichens indicate that they have evolved a successful strategy for survival within the lichen thallus (Honegger et al. 2013). Apart from being poorly understood for their diversity and ecology, the ELFs are still unexplored treasures for biomolecules which could lead to the development of novel drugs. More studies are needed with culture-dependent and culture-independent approaches to estimate the true diversity of ELFs. Furthermore, lichens in different environments must be explored to understand their distribution patterns, the influence by abiotic and biotic factors, and host recurrence of ELFs. The estimated diversity of ELFs is undocumented while many sequences deposited in GenBank typically provide limited taxonomic details. Furthermore, the interaction of ELF with the host lichenized fungi as well as with the co-occurring symbionts needs to be studied using modern techniques such as isotopologue profiling. This result will be useful to understand the cross-relationship between the different constituencies of the microbiome of lichens (Götz et al. 2010). The culture-independent techniques and high-throughput sequencing methods are helpful in taxonomic placement, understanding ecological role and evolutionary details of endophytes.

\section{Acknowledgments}

We are thankful to Director, CSIR-NBRI, Lucknow for providing laboratory facilities and partial financial support under project OLP101 and to Council of Scientific and Industrial Research, New Delhi, for financial support under JRF/SRF Fellowship. (CSIR-NBRI manuscript number CSIR-NBRI_MS/2020/08/02).

\section{References}

Arnold AE, Mejía LC, Kyllo D, Rojas EI et al. 2003 - Fungal endophytes limit pathogen damage in a tropical tree. Proceedings of the National Academy Science 100, 15649-15654.

Arnold AE, Miadlikowska J, Higgins KL. 2009 - Aphylogenetic estimation of trophic transition networks for ascomycetous Fungi: Are lichens cradles of symbiotrophic Fungal diversification? Syst Biol 58(3), 283-297.

Basnet BB, Chen B, Suleimen YM, Ma K et al. 2019a - Cytotoxic secondary metabolites from the endolichenic fungus Hypoxylon fuscum. Planta Medica 85 (13), 1088-1097. 
Basnet BB, Liu L, Chen B, Suleimen YM et al. 2019b - Four new cytotoxic arborinane-type triterpenes from the endolichenic fungus Myrothecium inundatum. Planta Medica 85 (09/10), 701-707.

Chagnon P, U'Ren JM, Miadlikowska J, Lutzoni F et al. 2016 - Interaction type influences ecological network structure more than local abiotic conditions: evidence from endophytic and endolichenic fungi at a continental scale. Oecologia 180, 181-191.

Chen GD, Chen Y, Gao H, Shen LQ et al. 2013 - Xanthoquinodins from the endolichenic fungal strain Chaetomium elatum. Journal of Natural Products 76, 702-709.

Chen GD, Bao YR, Huang YF, Hu D et al. 2014 - Three pairs of variecolortide enantiomers from Eurotium sp. with caspase-3 inhibitory activity. Fitoterapia 92, 252-259.

Chen M, Wang R, Zhao W, Yu L et al. 2019 - Isocoumarindole A, a chlorinated isocoumarin and indole alkaloid hybrid metabolite from an endolichenic fungus Aspergillus sp. Organic Letters 21(5), 1530-1533.

Dettki H, Esseen PA. 1998 - Epiphytic macrolichens in managed and natural forest landscapes: a comparison at two spatial scales. Ecographysiology 21, 613-624

Ding G, Li Y, Fu S, Liu S et al. 2009 - Ambuic acid and torreyanic acid derivatives from Endolichenic fungus Pestalotiopsis sp. Journal of Natural Products 72, 182-186.

Duo Y, Wang X, Jiang D, Wang H et al. 2014 - Metabolites from Aspergillus versicolor, an endolichenic fungus from the lichen Lobaria retigera. Drug discoveries \& Therapeutics 8 , 84-88.

Girlanda M, Isocrono D, Bianco C, Luppi-Mosca AM. 1997 - Two foliose lichens as microfungal ecological niches. Mycologia 89, 531-536.

Götz A, Eylert E, Eisenreich W, Goebel W. 2010 - Carbon metabolism of enterobacterial humn pathogens growing in epithelial colorectal adeno carcinoma (Caco-2) cells. PloS ONE. 5: e10586

Govinda Rajulu MB, Thirunavukkarasu N, Kumar SS, Kaur T et al. 2020 - Endolichenic fungal diversity associated with some lichens of Western Ghats. Plant Medica 86, 960-966.

Guo LD, Xu L, Zheng WH, Hyde KD. 2004 - Genetic variation of Alternaria alternata, an endophytic fungus isolated from Pinus tabulaeformis as determined by random amplified microsatellites (RAMS). Fungal Diversity 16, 53-65.

Hawksworth DL, Grube M. 2020 - Lichens redefined as complex ecosystems. New Phytologist. Doi 10.1111/nph.16630

He JW, Chen GD, Gao H, Yang F et al. 2012 - Heptaketides with antiviral activity from three endolichenic fungal strains Nigrospora sp. Alternaria sp. and Phialophora sp. Fototerapia 83, 1087-1091.

Honegger R. 1991 - Fungal evolution: symbiosis and morphogenesis. In Symbiosis as a Source of Evolutionary Innovation. Margulis, L., and Fester, R. (eds). Cambridge, MA, USA. The MIT Press 319-340.

Honegger R, Axe L, Edwards D. 2013 - Bacterial epibionts and endolichenic actinobacteria and fungi in the lower Devonian lichen Chlorolichenomycites salopensis. Fungal Biol 117, 512 518.

Hu CH, Zhou YH, Xie F, Li YL et al. 2017 - Two new $\alpha$-pyrone derivatives from an endolichenic fungus Tolypocladium sp. Journal of Asian Natural Products Research 19, 786-792.

Jayakumar K, Prasad BG, Charan TP, Swarana KG et al. 2016 - Biomedical potential of Talaromyces tratensis - A New endolichenic fungi associated with high altitude crustose lichen Lecanora sp. Research J. Pharma. Biol. Chem. Sci 7(5), 1412-1419.

Kannangara BTSDP, Rajapaksha RSCG, Paranagama PA. 2008 - Nature and bioactivities of endolichenic fungi in Pseudocyphellaria sp., Parmotrema sp. and Usnea sp. at Hakgala montane forest in Sri Lanka. Letters in applied microbiology 10(7), 3412-3420.

Kellogg JJ, Raja HA. 2016 - Endolichenic fungi: a new source of rich bioactive secondary metabolites on the horizon. Phytochemistry Reviews 16(2), 271-293. 
Kim KH, Beemelmanns C, Murillo C, Guillen A et al. 2014 - Naphthalenones and isocoumarins from a costarican fungus Xylariaceae sp. CR1546C. The Journal of Chemical Research 38, $722-725$.

Kim JW, Ko W, Kim E, Kim GS et al. 2018a - Anti-inflammatory phomalichenones from an endolichenic fungus Phoma sp. The Journal of Antibiotics 71, 753-756.

Kim TY, Jang NH, Chi WJ, Bae CH et al. 2018b - Nematicidal activity of grammicin produced by Xylaria grammica kctc 13121BP against Meloidogyne incognita. Pest Management Science 74, 384-391.

Li WC, Zhou J, Guo SY, Guo LD. 2007 - Endophytic fungi associated with lichens in Baihua Mountain of Beijing, China. Fungal Divers 25, 69-80.

Li G, Wang H, Zhu R, Sun L et al. 2012 - Phaerosphaerins A-F, cytotoxic perylenequinones from an endolichenic fungus Phaeosphaeria sp. Journal of Natural Products 75, 142-147.

Li XB, Zhou YH, Zhu RX, Chang WQ et al. 2015a - Identification and biological evaluation of secondary metabolites from the endolichenic fungus Aspergillus versicolor. Chemistry \& Biodiversity $12,575-592$.

Li XB, Li L, Zhu RX, Li W et al. 2015b - Tetramic acids and pyridine alkaloids from the endolichenic fungus Tolypocladium cylindrosporum. Journal of Natural Products 78, 21552160.

Li W, Gao W, Zhang M, Li YL et al. 2016 - p-Terphenyl derivatives from the endolichenic fungus Floricola striata. Journal of Natural Products 79, 2188-2194.

Li Y, Zhu R, Zhang J, Xie F et al. 2018 - Ophiosphaerellins A-I, polyketide-derived compounds from the endolichenic fungus Ophiosphaerella korrae ACS Omega 3, 176-180.

Li YL, Zhu RX, Li G, Wang NN et al. 2019 - Secondary metabolites from the endolichenic fungus Ophiosphaerella korrae. RSC Advances 9, 4140-4149.

Lücking R, Hodkinson BP, Leavitt SD. 2017 - The 2016 classification of lichenized fungi in the Ascomycota and Basidiomycota - approaching one thousand genera. Bryologist 119, 361416.

Maduranga K, Attanayake RN, Santhirasegaram S, Weerakoon G et al. 2018 - Molecular phylogeny and bioprospecting of Endolichenic Fungi (ELF) inhabiting in the lichens collected from a mangrove ecosystem in Sri Lanka. PLoS ONE 13(8), e0200711.

Mark K, Laanisto L, Bueno CG, Niinemets Ü et al. 2020 - Contrasting co-occurrence patterns of photobiont and cystobasidiomycete yeast associated with common epiphytic lichen species. New Phytologist 227(5), 1362-1375.

Miadlikowska J, Arnold AE, Lutzoni F. 2004 - Diversity of cryptic fungi inhabiting healthy lichen thalli in a temperate and tropical forest. Ecol. Soc. 89, 349-350.

Oh SY, Yang JH, Woo JJ, Oh SO et al. 2020 - Diversity and distribution patterns of Endolichenic Fungi in Jeju Island, South Korea. Sustainability 12, 3769.

Padhi S, Tayung K. 2015 - In vitro antimicrobial potentials of endolichenic fungi isolated from thalli of Parmelia lichen against some human pathogens. Beni-Suef University Journal of Basic and Applied Sciences 4(4), 299-306.

Padhi S, Das D, Panja S, Tayung K. 2016 - Molecular characterization and antimicrobial activity of endolichenic fungus Aspergillus sp. isolated from Parmelia caperata of Similipal Biosphere Reserve, India. Interdiscip. Sci. 9(2), 237-246.

Padhi S, Masi M, Cimmino A, Tuzi A et al. 2019 - Funiculosone, a substituted dihydroxanthene1,9-dione with two of its analogues produced by an endolichenic fungus Talaromyces funiculoses and their antimicrobial activity. Phytochemistry 157, 175-183.

Paranagama PA, Wijeratne EMK, Burns AM, Marron MT et al. 2007 - Heptaketides from Corynespora sp. inhabiting the cavern beard lichen, Usnea cavernosa: first report of metabolites of an endolichenic fungus. J Nat Prod 70, 1700-1705.

Petrini O, Hake U, Dreyfuss MM. 1990 - An analysis of fungal communities isolated from fruticose lichens. Mycologia 82, 444-451. 
Prateeksha, Bajpai R, Yusuf MA, Upreti DK et al. 2020 - Endolichenic fungus, Aspergillus quandricinctus of Usnea longissima inhibits quorum sensing and biofilm formation of Pseudomonas aeruginosa PAO1. Microbial pathogenesis 140, 103933.

Rashmi M, Kushveer JS, Sarma VV. 2019 - A worldwide list of endophytic fungi with notes on ecology and diversity. Mycosphere 10(1), 798-1079.

Samanthi KAU, Wickramarachchi S, Wijeratne EMK, Paranagama PA. 2015a - Two new bioactive polyketides from Curvularia trifolii, an endolichenic fungus isolated from Usnea sp., in Sri Lanka. Journal of the National Science Foundation Sri Lanka 43 (3), 217-224.

Samanthi KAU, Wickramaarachchi S, Wijeratne E, Paranagama P. 2015b - Two new antioxidant active polyketides from Penicillium citrinum an endolichenic fungus isolated from Parmotrema sp. in Sri Lanka. Journal of the National Science Foundation of Sri Lanka 43(2), 119-126.

Sarma P, Dkhar MS, Kayang H, Kumar M et al. 2020 - Diversity of endophytic fungi associated with Hedychium spicatum Ham ex $\mathrm{Sm}$. and their antifungal activity against the phytopathogen Alternaria solani. Studies in Fungi 5(1), 84-93.

Spribille T, Tuovinen V, Resl P, Vanderpool D et al. 2016 - Basidiomycete yeasts in the cortex of ascomycete macrolichens. Science 353, 488-492.

Stone JK, Polishook JD, White JF. 2012 - Endophytic fungi. In: Encyclopedia of Science and Technology, McGraw-Hill Education, USA. 241-270.

Suryanarayanan TS, Thennarasan S. 2004 - Temporal variation in endophyte assemblages of Plumeria rubra leaves. Fungal Diversity 15, 197-204

Suryanarayanan TS, Thirunavukkarasu N, Hariharan GN, Balaji P. 2005 - Occurrence of nonobligate inside lichen thalli. Sydowia 57, 120-130.

Suryanarayanan TS, Govinda Rajulu MB, Rajamani T, Tripathi M et al. 2017 - Endolichenic fungi in lichens of Champawat district, Uttarakhand, Northern India. Mycol Prog 16, 205-211.

Tripathi M, Gupta RC, Joshi Y. 2014 - Assessment of endolichenic fungal diversity in some forests of Kumaun Himalaya. Current Science. 107(5), 745-748.

Tripathi M, Joshi Y. 2019 - Taxonomic Descriptions of Endolichenic Fungi. In: Endolichenic Fungi: Present and Future Trends. Springer, Singapore.

U'Ren JM, Lutzoni F, Miadlikowska J, Laetsch AD, Arnold AE. 2012 - Host and geographic structure of endophytic and endolichenic fungi at a continental scale. American Journal of Botany 99(5), 898-914.

Vinayaka K, Krishnamurthy YL, Shivakumar B, Prashith KTR. 2016 - Association and variation of Endophytic fungi among some Macrolichens in Central Western Ghats, Southerns India. International Journal of Current Microbiology \& Applied Sciences. 5(6), 115-124.

Wang Y, Niu S, Liu S, Guo L et al. 2010 - The first naturally occurring thiepinols and thienol from an endolichenic fungus Coniochaeta sp. Organic Letters 12, 5081-5083.

Wang QX, Bao L, Yang XL, Guo H et al. 2012- Polyketides with antimicrobial activity from the solid culture of endolichenic fungus Ulocladium sp. Fitoterapia 83, 209-214.

Wang Y, Zheng Y, Wang X, Wei X, Wei J. 2016 - Lichen associated fungal community in Hypogymnia hypotrypa (Parmeliaceae, Ascomycota) affected by geographic distribution and altitude. Front Microbiol 7, 1231.

Wang H, Umeokoli BO, Eze P, Heering C et al. 2017 - Secondary metabolites of lichen associated fungus Apiospora montagnei. Tetrahedron Letters, 58, 1702-1705.

Wijayawardene NN, Hyde KD, Al-Ani LKT, Tedersoo L et al. 2020 - Outline of fungi and fungus like-taxa. Mycosphere 11(1), 1060-1456.

Wijeratne EK, Bashyal BP, Liu MX, Rocha DD et al. 2012 - Geopyxins A-E, ent-kaurane diterpenoids from endolichenic fungal strains Geopyxis aff. Majalis and Geopyxis sp. AZ0066: structure-activity relationships of geopyxins and their analogues. Journal of Natural Products 75, 361-369.

Wijeratne EK, Gunaherath GKB, Chapla VM, Tillotson J et al. 2016 - Oxaspirol b with p97 inhibitory activity and other oxaspirols from Lecythophora sp. FL1375 and FL1031, 
Endolichenic fungi inhabiting Parmotrema tinctorum and Cladonia evansii. Journal of Natural Products 79, 340-352.

Wu W, Dai H, Bao L, Lu J et al. 2011 - Isolation and structural elucidation of proline-containing cyclopentapeptides from an endolichenic Xylaria sp. Journal of Natural Products 74, 13031308.

Wu YH, Chen GD, Wang CX, Hu D et al. 2015 - Pericoterpenoid A, a new bioactive cadinanetype sesquiterpene from Periconia sp. Journal of Asian Natural Products Research 17(6), 671-675.

Xie F, Chang W, Zhang M, Li Y et al. 2016 - Quinone derivatives isolated from the endolichenic fungus Phialocephala fortinii are MDR1 modulators that combat azole resistance in Candida albicans. Scientific Reports 6, 33687.

Ye F, Chen GD, He JW, Li XX et al. 2013 - Xinshengin, the first altenusin with tetracyclic skeleton core from Phialophora spp. Tetrahedron Letters 54, 4551-4554.

Yuan C, Wang HY, Wu CS, Jiao Y et al. 2013 - Austdiol, fulvic acid and citromycetin derivatives from an endolichenic fungus, Myxotrichum sp. Phytochemistry Letters 6, 662-666.

Yuan C, Ding G, Wang HY, Guo YH et al. 2017 - Polyketide-terpene hybrid metabolites from an endolichenic fungus Pestalotiopsis sp. BioMed Research International 10 Article ID 6961928 pages.

Yuan WH, Teng MT, Sun SS, Ma L et al. 2018 - Active metabolites from endolichenic fungus Talaromyces sp. Chemistry \& Biodiversity 5, e1800371

Yuan X, Xiao S, Taylor TN. 2005 - Lichen-like symbiosis 600 million years ago. Science 308, 1017-1020.

Yu NH, Park SY, Kim JA, Park CH et al. 2018 - Endophytic and endolichenic fungal diversity in maritime Antarctica based on cultured material and their evolutionary position among Dikarya. Fungal Systematic Evolution 22, 263-272.

Zhang F, Liu S, Lu X, Guo L et al. 2009 - Allenyl and alkynyl phenyl ethers from endolichenic fungus Neurospora terricola. Journal of Natural Products 72, 1782-1785.

Zhang ZB, Zeng QG, Yan RM, Wang Y et al. 2011 - Endophytic fungus Cladosporium cladosporioides LF70 from Huperzia serrata produces Huperzine A. World J Microbiol Biotechnol 27, 479-486.

Zhang F, Li L, Niu S, Si Y et al. 2012 - A thiopyranchromenone and other chromone derivatives from an endolichenic fungus, Preussia africana. Journal of Natural Products 75, 230-237.

Zhang T, Wei XL, Wei YZ, Liu HY, Yu LY. 2016 - Diversity and distribution of cultured endolichenic fungi in the Ny-Ålesund Region, Svalbard (High Arctic). Sci Rep 20, 461-470.

Zhao H, Wang GQ, Tong XP, Chen GD et al. 2014 - Diphenyl ethers from Aspergillus sp. and their anti-Ab42 aggregation activities. Fitoterapia 98, 77-83.

Zhao Q, Wang CX, Yu Y, Wang GQ et al. 2015 - Nodulisporipyrones A-D, new bioactive $\alpha$ pyrone derivatives from Nodulisporium sp. Journal of Asian Natural Products Research 17, 567-575.

Zheng QC, Kong MZ, Zhao Q, Chen GD et al. 2014 - Chaetoglobosin Y, a new cytochalasan from Chaetomium globosum. Fototerapia 93, 126-131.

Zhou YH, Zhang M, Zhu RX, Zhang JZ et al. 2016 - Heptaketides from an endolichenic fungus Biatriospora sp. and their antifungal activity. Journal of Natural Products 79, 2149-2157. 